\title{
Ergodic non-abelian smooth extensions of an irrational rotation
}

\author{
Julien Brémont
}

\section{Abstract}

Above an irrational rotation on the Circle, we build optimally smooth ergodic cocycles with values in some nilpotent or solvable subgroups of triangular matrices.

\section{Introduction}

Let $(X, \mathcal{B}, T, \mu)$ be an invertible dynamical system, where $(X, \mathcal{B})$ is a standard Borel space, $T$ an invertible measurable transformation of $X$ and $\mu$ a $T$-invariant ergodic Borel probability measure. Let $G$ be a locally compact separable group. We suppose that the topology on $G$ is defined by some fixed metric and we write $V_{\varepsilon}(c)$ for the open ball of radius $\varepsilon>0$ and center $c \in G$. In the sequel $G$ is $\mathbb{R}^{d}$ or a closed subgroup of $G L_{d}(\mathbb{R})$.

To a measurable function $\varphi: X \rightarrow G$ one canonically associates the cylindrical or skew-product transformation $T_{\varphi}:(x, g) \longmapsto(T x, \varphi(x) g)$ on $X \times G$. Iterates of $T_{\varphi}$ involve the cocycle $\left(\varphi_{n}\right)_{n \in \mathbb{Z}}$ :

$$
\varphi_{n}(x)=\left\{\begin{array}{cc}
\varphi\left(T^{n-1} x\right) \cdots \varphi(x), & n>0 \\
e, & n=0 \\
\varphi^{-1}\left(T^{n} x\right) \cdots \varphi^{-1}\left(T^{-1} x\right), & n<0 .
\end{array}\right.
$$

We next use the notation $T \varphi$ for $\varphi \circ T$. When $G$ is abelian, the law in $G$ is written additively and $\varphi_{n}$ is then the Birkhoff $\operatorname{sum} \varphi_{n}(x)=\sum_{k=0}^{n-1} \varphi\left(T^{k} x\right)$, for $n \geq 1$.

Fixing a left-invariant Haar measure $\lambda$ on $G$, the product measure $\mu \otimes \lambda$ on $X \times G$ is $T_{\varphi}$-invariant. We consider in this article the existence and the construction of $\varphi$ such that $T_{\varphi}$ is ergodic for $\mu \otimes \lambda$. In the whole text, the ergodicity of $T_{\varphi}$ only refers to $\mu \otimes \lambda$. Such $\varphi$ provide ergodic dynamical systems, of particular interest when $G$ is non-compact since the invariant measure is infinite.

The existence of $\varphi: X \rightarrow G$ with $T_{\varphi}$ ergodic requires that $G$ is amenable, cf Golodets and Sinel'shchikov [9]. A problem is then to exhibit concrete examples and a large part of the literature on cocycles is devoted to expliciting classes of $\varphi: X \rightarrow G$ such that $T_{\varphi}$ is ergodic for specific groups and dynamical systems. The case when $G=\mathbb{R}, X$ is the Circle $\mathbb{T}^{1}=\mathbb{R} \backslash \mathbb{Z}$ and $T$ is an irrational rotation $R_{\alpha}$ of angle $\alpha$ has received considerable attention, see for instance the bibliography in Conze [4]. Most examples are discontinuous and piecewise smooth. A non-commutative situation has recently been considered by Greschonig [10], when $(X, T)=\left(\mathbb{T}^{1}, R_{\alpha}\right)$ and $G$ is the discrete Heisenberg group $N_{3}(\mathbb{Z})$. An ergodic BV example is built, using linear combinations of interval indicator functions, for any $\alpha$ with bounded partial quotients.

When $(X, T)$ and $G$ have a smooth structure, it is natural to ask for smooth examples. For a large class of topological dynamical systems and amenable groups, Nerurkar [15] has first given genericity results. More precisely, among continuous $\varphi$, the ones for which $T_{\varphi}$ is ergodic are generic in the Baire Category sense. Producing $\varphi: X \rightarrow G$ with an a priori smoothness so that $T_{\varphi}$ is ergodic is often delicate and can be impossible. In the situation when $(X, T)$ admits fast periodic approximations, Nerurkar [16] proved genericity results on ergodic cocycles with high regularity. For $G=\mathbb{R}$ and $(X, T)=\left(\mathbb{T}^{1}, R_{\alpha}\right)$, existence results, based on tower constructions, about ergodic cocycles with optimal smoothness and other properties are contained in Volný [20].

Let us now describe the content of this article. We fix the base to $(X, T)=\left(\mathbb{T}^{1}, R_{\alpha}\right)$. When $G=\mathbb{R}^{d}$ and for a general $\alpha$ with unbounded partial quotients, the optimal regularity of $\varphi: \mathbb{T}^{1} \rightarrow \mathbb{R}^{d}$ 
with $T_{\varphi}$ ergodic is $\mathcal{C}^{1}$, since $\mathcal{C}^{1+\varepsilon}$-extensions are not ergodic if $\alpha$ has Diophantine type 1 . This is recalled below. The condition on $\alpha$ for a $\mathcal{C}^{1}$ regularity is minimal, since ergodic $\mathcal{C}^{1}$-extensions are impossible if $\alpha$ has bounded partial quotients.

The purpose of this paper is to prove similar results in some non-abelian situations. More precisely, under the same hypothesis on $\alpha$, we build $\mathcal{C}^{1}$ ergodic cocycles in the following closed subgroups of the group of invertible $d \times d$ upper-triangular matrices :

- the nilpotent non-abelian group $N_{d}(\mathbb{R})$ of matrices with unit diagonal elements, when $d \geq 3$,

- the solvable non-nilpotent group $\operatorname{Sol}_{3}(\mathbb{R})$, when $d=3$.

In these cases, the optimal regularity of an ergodic extension is thus the same as for $\mathbb{R}^{d}$. Our strategy is semi-abstract and therefore essentially furnishes existence results. We develop via elementary Fourier Analysis a general method for building smooth ergodic cocycles by explicit formulas in terms of the sequence of convergents of $\alpha$.

\section{Preliminaries}

We first introduce the notion of essential value, used for considering the ergodicity of a skewproduct. We next present the basic stone with which our examples are built and, in a first application, focus on the situation when $G=\mathbb{R}^{d}$.

\subsection{Essential values; a general lemma for abelian groups}

In the general context of the beginning of the introduction, the question of the ergodicity of a skew-product can be reformulated using essential values. See Schmidt [17] or Feldman-Moore [7]. Let $\varphi: X \rightarrow G$ be measurable.

Definition 2.1. An element $c \in G \cup\{\infty\}$ is an essential value for $\varphi$, if for any $\varepsilon>0$ and Borel set $A \subset X$ with $\mu(A)>0$, there is $n \in \mathbb{Z}$ so that $\mu\left(A \cap T^{-n} A \cap\left\{\varphi_{n} \in V_{\varepsilon}(c)\right\}\right)>0$.

The set of finite essential values is a closed subgroup of $G$, denoted by $\mathcal{E}(\varphi)$ in the sequel. The skew-product $T_{\varphi}$ on $X \times G$ is then ergodic if and only if $\mathcal{E}(\varphi)=G$.

When $G$ is abelian, the non-degeneracy of the cocycle along a rigid sequence provides finite essential values. Minimal translations on a torus furnish a natural situation for this to occur.

The following lemma is prop 9 in Lemańczyk-Parreau-Volný [13]. See also the notion of quasi-period in [4]. Reordering arguments appearing in the literature, we give another proof.

LEMMA 2.2.

Let $G$ be abelian and $\varphi: X \rightarrow G$ be measurable. If $\left(k_{n}\right) \rightarrow+\infty$ verifies $T^{k_{n}} x \rightarrow x, \mu-$ ae, and the law of $\varphi_{k_{n}}$ converges to a measure $\nu$ for the vague topology, then $\operatorname{Supp}(\nu) \subset \mathcal{E}(\varphi)$.

Proof of the lemma :

Starting as in [4], for a Borel set $A$ :

$$
\int_{X}\left|1_{A}-1_{T^{-k_{n}} A}\right| d \mu \longrightarrow_{n \rightarrow+\infty} 0 .
$$

Indeed, this is true when $1_{A}$ is replaced by a continuous function. This property is extended to functions in $L^{1}(\mu)$ via the invariance of the measure $\mu$.

Following next [17], if $F$ is compact and such that $F \cap \mathcal{E}(\varphi)=\varnothing$, then for all $B$ with $\mu(B)>0$, there is $A \subset B$ with $\mu(A)>0$ such that for all $n \in \mathbb{Z}, \mu\left(A \cap T^{-n} A \cap\left\{\varphi_{n} \in F\right\}\right)=0$. This is where the abelian character of $G$ is used. By (2.1), we get $\mu\left(A \cap\left\{\varphi_{k_{n}} \in F\right\}\right) \rightarrow 0$, as $n \rightarrow+\infty$.

Via the exhaustion lemma 1.0.7 in Aaronson [1] , we obtain $X=\cup_{l \geq 1} A_{l}$ modulo $\mu$, where $\mu\left(A_{l} \cap\right.$ $\left.\left\{\varphi_{k_{n}} \in F\right\}\right) \rightarrow 0$ for each $l \geq 1$. Therefore : 


$$
\mu\left\{\varphi_{k_{n}} \in F\right\} \rightarrow_{n \rightarrow+\infty} 0 .
$$

Suppose then that $c \in S u p p(\nu) \backslash \mathcal{E}(\varphi)$, where $\nu$ is the vague limit of the laws of $\varphi_{k_{n}}$. Since $\mathcal{E}(\varphi)$ is closed, there is $\varepsilon>0$ such that $V_{\varepsilon}(c) \cap \mathcal{E}(\varphi)=\varnothing$. Fix a continuous $\psi: G \rightarrow[0,1]$ so that $\psi>0$ on $V_{\varepsilon / 4}(c)$ and $\psi=0$ outside $V_{\varepsilon / 2}(c)$. By $(2.2), \mu\left\{\varphi_{k_{n}} \in \bar{V}_{\varepsilon / 2}(c)\right\} \rightarrow 0$. However :

$$
\mu\left\{\varphi_{k_{n}} \in \bar{V}_{\varepsilon / 2}(c)\right\} \geq \int_{X} \psi\left(\varphi_{k_{n}}\right) d \mu \rightarrow \int_{G} \psi d \nu>0 .
$$

This contradiction concludes the proof of the lemma.

Remark - A sequence $\left(k_{n}\right)$ as in the statement of the lemma is said "rigid". Recall that for the vague topology a sequence of probability measures is relatively compact among Borel measures with mass less than or equal to one.

In the sequel, except for section $2.5, X$ is the torus $\mathbb{T}^{1}$ identified to $\mathbb{R} / \mathbb{Z}$, with an irrational rotation $T$ of angle $\alpha$ and Lebesgue measure $\mu$.

\subsection{Squashability}

In most cases, we also examine the squashability of $\left(X \times G, T_{\varphi}\right)$. This question comes after ergodicity. Following Aaronson [1, 2], an infinite ergodic dynamical system $(Y, S, \nu)$ is squashable, if there exists a non-singular non-measure-preserving transformation $Q$ commuting with $S$. In this case, since $\nu \circ Q^{-1}$ is $S$-invariant and equivalent to $\nu$, there is a constant $c$ such that $\nu \circ Q^{-1}=$ $c \nu$, with therefore $c \neq 1$. The dynamical system $(Y, S, \nu)$ is completely squashable if any $c \neq 1$ is possible.

Squashability is an obstruction to the existence of a generalised law of large numbers, ie of a measurable $\Psi:\{0,1\}^{\mathbb{N}} \rightarrow \mathbb{R}_{+}$such that $\nu(A)=\Psi\left(\left(1_{A}\left(S^{n} \omega\right)\right)_{n>1}\right), \nu-a e$, for all measurable set $A$. Indeed, reproducing the proof in $[\mathbf{1}]$, take $A$ with positive finite measure and $\omega$ such that $\omega$ and $Q \omega$ are $\nu$-typical. A contradiction is obtained as follows :

$$
\nu(A)=\Psi\left(\left(1_{A}\left(S^{n} Q \omega\right)\right)\right)=\Psi\left(\left(1_{A}\left(Q S^{n} \omega\right)\right)\right)=\Psi\left(\left(1_{Q^{-1} A}\left(S^{n} \omega\right)\right)\right)=\nu\left(Q^{-1} A\right)=c \nu(A) .
$$

In our setting we have the following result from [1] (proposition 8.4.1) :

\section{Proposition 2.3.}

Let $X=\mathbb{T}^{1}$ with an irrational rotation $T$ of angle $\alpha$ and Lebesgue measure $\mu$. Let $G$ be a locally compact second countable group and $\varphi: X \rightarrow G$ be measurable. If $T_{\varphi}$ is ergodic and $Q: X \times G \rightarrow$ $X \times G$ is non-singular with $Q \circ T_{\varphi}=T_{\varphi} \circ Q$, then there exist $\beta \in \mathbb{R}$, a continuous surjective group endomorphism $w: G \rightarrow G$ and a measurable $f: X \rightarrow G$ so that :

$$
Q(x, y)=(x+\beta, f(x) w(y)) \text { and } \varphi(x+\beta)=T f(x) w(\varphi(x)) f^{-1}(x),
$$

for all $x \in X, y \in G$.

The proof in [1] is given when $G$ is abelian. Using the notations of [1], it remains valid in general when taking the translations $Q_{g}(x, y)=(x, y g)$ to show that $F^{-1} . F \circ Q_{g}$ is $T_{\varphi}$-invariant.

\subsection{Smooth ergodic cocycles in $\mathbb{R}^{d}$}

In the present paragraph $G=\mathbb{R}^{d}$. The sequence of convergents of $\alpha$ is denoted by $\left(p_{n} / q_{n}\right)$ and the partial quotients by $\left(a_{n}\right)$. Recall that they obey the recursive relations : 


$$
\left\{\begin{array}{c}
p_{n+1}=a_{n+1} p_{n}+p_{n-1} \text { and } q_{n+1}=a_{n+1} q_{n}+q_{n-1}, n \geq 0 \\
p_{-1}=1, p_{0}=0, q_{-1}=0, q_{0}=1
\end{array}\right.
$$

We say $\alpha$ has bounded partial quotients (bpq), if $a_{n} \leq C$ for some constant $C>0$. We write $((x))$ for the distance from $x$ to $\mathbb{Z}$. Recall also that $1 /\left(q_{n}+q_{n+1}\right) \leq\left(\left(q_{n} \alpha\right)\right)<1 / q_{n+1}$.

The constructions that appear in this article are essentially variations on a single example. Consider positive integers $\left(u_{k}\right)_{k \geq 1}$ with $\sum 1 / u_{k}<\infty$ and a subsequence $\left(v_{k}\right)_{k \geq 1}$ of the $\left(q_{n}\right)$. Define :

$$
f(x)=\sum_{k \geq 1} \frac{1}{u_{k}} \sin \left(2 \pi v_{k} x\right) .
$$

Introducing a sequence of times $\left(t_{n}\right)$, we compute $f_{t_{n}}$ :

$$
\begin{aligned}
f_{t_{n}}(x)=\operatorname{Im} \sum_{k \geq 1} \frac{e^{2 i \pi v_{k} x}}{u_{k}}\left(\frac{e^{2 i \pi v_{k} t_{n} \alpha}-1}{e^{2 i \pi v_{k} \alpha}-1}\right) & =\sum_{k \geq 1} \frac{\sin \left(\pi v_{k} t_{n} \alpha\right)}{u_{k} \sin \left(\pi v_{k} \alpha\right)} \sin \left[\pi v_{k}\left(2 x+\left(t_{n}-1\right) \alpha\right)\right] \\
& =\sum_{k<n}+\sum_{k=n}+\sum_{k>n}=A_{n}+B_{n}+C_{n} .
\end{aligned}
$$

An existence result, classical when $d=1$, is the following :

Proposition 2.4.

i) Let $\alpha$ have non bpq. Choose a strictly increasing sequence $(\theta(k))_{k \geq 1}$ so that $a_{\theta(k)+1} \geq k^{3}$. Set :

$$
f=\left(f^{i}\right)_{1 \leq i \leq d}, \text { where } f^{i}(x)=\sum_{k \geq 1} \frac{\sin \left(2 \pi q_{\theta(d k+i)} x\right)}{k^{2} q_{\theta(d k+i)}} .
$$

Then $f$ is $\mathcal{C}^{1}$ and $T_{f}: \mathbb{T}^{1} \times \mathbb{R}^{d} \rightarrow \mathbb{T}^{1} \times \mathbb{R}^{d}$ is ergodic and non-squashable.

ii) Let $\alpha$ have bpq. Fix a decreasing function $\varepsilon$ such that $\varepsilon(h) \rightarrow+\infty$, as $h \rightarrow 0^{+}$, and choose a strictly increasing sequence $(\theta(k))_{k \geq 1}$ satisfying $\theta(k) \geq k^{2}$ and $\varepsilon\left(1 / q_{\theta(k)}\right) \geq k$. Set :

$$
f=\left(f^{i}\right)_{1 \leq i \leq d}, \text { where } f^{i}(x)=\sum_{k \geq 1} \frac{\sin \left(2 \pi q_{\theta(d k+i)} x\right)}{q_{\theta(d k+i)}} .
$$

Then $h \varepsilon(h)$ is a modulus of continuity for $f$ and $T_{f}: \mathbb{T}^{1} \times \mathbb{R}^{d} \rightarrow \mathbb{T}^{1} \times \mathbb{R}^{d}$ is ergodic and nonsquashable.

Proof of the proposition :

$i)$ We fix $1 \leq j \leq d$ and consider the rigid sequence $\left(t_{j, n}\right)_{n \geq 1}=\left(n^{2} q_{\theta(d n+j)}\right)_{n \geq 1}$. For each $1 \leq i \leq d$, we focus on the decomposition $\left(A_{n}^{i}, B_{n}^{i}, C_{n}^{i}\right)$ associated to $f^{i}$ and given by $(2.3)$.

Using that $q_{\theta(k+1)} / q_{\theta(k)} \rightarrow+\infty$, as $k \rightarrow+\infty$, we have for a generic constant $C>0$ :

$$
\begin{aligned}
& \left|A_{n}^{i}\right| \leq C \sum_{k<n} \frac{n^{2}\left(\left(q_{\theta(d n+j)} \alpha\right)\right)}{k^{2}\left(\left(q_{\theta(d k+i)} \alpha\right)\right)} \leq C \frac{n^{2} q_{\theta(d(n-1)+i)+1}}{q_{\theta(d n+j)+1}} \leq C \frac{n^{2}}{a_{\theta(d n+j)+1}} \rightarrow_{n \rightarrow+\infty} 0, \\
& \left|C_{n}^{i}\right| \leq C \sum_{k>n} \frac{n^{2} q_{\theta(d n+j)}}{k^{2} q_{\theta(d k+i)}} \leq C \frac{q_{\theta(d n+j)}}{q_{\theta(d(n+1)+i)}} \leq \frac{C}{a_{\theta(d n+j)+1}} \rightarrow_{n \rightarrow+\infty} 0,
\end{aligned}
$$

due to the assumption on $\theta$ and since in each case the argument of $\theta$ in the numerator is strictly less than that in the denominator. Next $B_{n}^{i}=w_{n}^{i} \sin \left[\pi q_{\theta(d n+i)}\left(2 x+\left(t_{j, n}-1\right) \alpha\right)\right]$, with the expression : 


$$
w_{n}^{i}=\frac{\sin \left(\pi n^{2} q_{\theta(d n+j)} q_{\theta(d n+i)} \alpha\right)}{n^{2} q_{\theta(d n+i)} \sin \left(\pi q_{\theta(d n+i)} \alpha\right)} \sim_{n \rightarrow+\infty} \varepsilon_{n}(i, j) \frac{\pi n^{2} q_{\theta(d n+\min (i, j))}\left(\left(q_{\theta(d n+\max (i, j))} \alpha\right)\right)}{\pi n^{2} q_{\theta(d n+i)}\left(\left(q_{\theta(d n+i)} \alpha\right)\right)},
$$

where $\varepsilon_{n}(i, j)= \pm 1$. The point is that $\left|w_{n}^{i}\right| \rightarrow 1$ if $i=j$ and $w_{n}^{i} \rightarrow 0$ otherwise. As a result, if $X$ is a random variable with uniform law on $[0,1]$ :

$$
f_{t_{j, n}} \rightarrow_{n \rightarrow \infty}\left(1_{i=j} \sin (2 \pi X)\right)_{1 \leq i \leq d} \text {, in law. }
$$

Lemma 2.2 then implies that $\{0\}^{j-1} \times[-1,1] \times\{0\}^{d-j} \subset \mathcal{E}(f)$. Since this holds for all $1 \leq j \leq d$, we obtain $\mathcal{E}(f)=\mathbb{R}^{d}$ and thus $T_{f}$ is ergodic.

If $T_{f}$ is squashable, by proposition 2.3 there exists a $d$-square invertible matrix $M$ satisfying $|\operatorname{det} M| \neq 1$, a measurable $\psi: \mathbb{T}^{1} \rightarrow \mathbb{R}^{d}$ and $\beta$ such that for almost-every $x \in \mathbb{T}^{1}$ :

$$
g(x):=M f(x)-f(x+\beta)=\psi(x)-\psi(x+\alpha) .
$$

Fix $1 \leq j \leq d$ and consider $\left(k_{n}\right)$ so that $q_{\theta\left(d k_{n}+j\right)} \beta \rightarrow \zeta_{j} \bmod (1)$ and $w_{k_{n}}^{j} \rightarrow \delta \in\{ \pm 1\}$. Then :

$$
\left(f_{t_{j, k_{n}}}(.), f_{t_{j, k_{n}}}(.+\beta)\right) \rightarrow_{n \rightarrow+\infty} \delta\left(1_{i=j}\left(\sin (2 \pi X), \sin \left(2 \pi\left(X+\zeta_{j}\right)\right)\right)\right)_{1 \leq i \leq d}, \text { in law. }
$$

Writing $\left(e_{i}\right)_{1 \leq i \leq d}$ for the canonical basis of $\mathbb{R}^{d}$, by lemma 2.2 , for all $c \in[0,1]$ :

$$
\delta \sin (2 \pi c) M e_{j}-\delta \sin \left(2 \pi\left(c+\zeta_{j}\right)\right) e_{j} \in \mathcal{E}(g)
$$

Since $\mathbb{R}^{d}$ is abelian and $g$ is a $T$-coboundary, we have $\mathcal{E}(g)=\{0\}$, cf [17]. As a result $M e_{j}$ is proportional to $e_{j}$ and $M_{j j} \sin (2 \pi c)=\sin \left(2 \pi\left(c+\zeta_{j}\right)\right)$, for all $c \in[0,1]$. Necessarily, $M_{j j}= \pm 1$. Since this holds for all $1 \leq j \leq d, M$ is diagonal and $\operatorname{det} M= \pm 1$, but this is a contradiction.

ii) Concerning the regularity of $f^{i}$, let $h>0$ be small so that $1 / q_{\theta(d l+i+1)} \leq h<1 / q_{\theta(d l+i)}$, for some $l \geq 1$. Splitting $f(x)-f(x+h)$ into $\sum_{k \leq l}$ and $\sum_{k>l}$, we obtain for a generic constant $C$ :

$$
|f(x)-f(x+h)| \leq C(h l+h) \leq C h l \leq C h \varepsilon\left(1 / q_{\theta(d l+i)}\right) \leq C h \varepsilon(h) .
$$

For the ergodicity and the squashability of $T_{f}$ we run the same proof with the sequence of times $\left(t_{j, n}\right)_{n \geq 1}=\left(q_{\theta(d n+j)}\right)_{n \geq 1}$, where $1 \leq j \leq d$ is fixed. Since $q_{\theta(k+1)} / q_{\theta(k)} \rightarrow+\infty$, as $k \rightarrow+\infty$, we only have to focus on the new $\left(w_{n}^{i}\right)_{1 \leq i \leq d}$ given by :

$$
w_{n}^{i}=\frac{\sin \left(\pi q_{\theta(d n+j)} q_{\theta(d n+i)} \alpha\right)}{q_{\theta(d n+i)} \sin \left(\pi q_{\theta(d n+i)} \alpha\right)} .
$$

As in (2.4), $w_{n}^{i} \rightarrow_{n \rightarrow+\infty} 0$, when $i \neq j$. Since $\alpha$ has bpq, let $M$ check $a_{n} \leq M$ for all $n \geq 1$. Using that $q_{l+1} \leq(M+1) q_{l}$, we have $q_{n}\left(\left(q_{n} \alpha\right)\right) \in[1 /(M+2), 1 /(1+1 /(M+1))]$. As a result :

$$
\frac{1}{\pi} \sin \left(\frac{\pi}{M+2}\right) \leq \liminf \left|w_{n}^{j}\right| \leq \limsup \left|w_{n}^{j}\right| \leq \frac{(M+2)}{\pi} .
$$

Consequently, $\left(w_{n}^{j}\right)_{n \geq 1}$ converges to a non-zero constant along a subsequence $\left(k_{n}\right)$. The reasoning is then the same, using this time the sequence $\left(t_{j, k_{n}}\right)$. This completes the proof of the proposition.

Remark 1 - Results related to item $i$ ) when $d=1$ can be found in [20], where the method is developed in order to produce completely squashable examples. In the context of $i i$ ), for instance, Liardet and Volný [14] have constructed ergodic cocycles with an absolutely continuous $f$.

Remark 2 - When $\left(\mathbb{T}^{1}, T\right)$ admits fast periodic approximations, in the spirit of $[\mathbf{1 6}]$ or $[\mathbf{2 0}]$, then the function $f: \mathbb{T}^{1} \rightarrow \mathbb{R}^{d}$ can be taken more regular : 
- of class $\mathcal{C}^{p}$, if $\lim \sup q_{n+1} / q_{n}^{p}=+\infty$, for some integer $p \geq 1$. Indeed, choose $(\theta(k))$ with $q_{\theta(k)+1} \geq k^{3} q_{\theta(k)}^{p}$ and define :

$$
f=\left(f^{i}\right)_{1 \leq i \leq d}, \text { with } f^{i}(x)=\sum_{k \geq 1} k^{-2} q_{\theta(d k+i)}^{-p} \sin \left(2 \pi q_{\theta(d k+i)} x\right) .
$$

Then $T_{f}: \mathbb{T}^{1} \times \mathbb{R}^{d} \rightarrow \mathbb{T}^{1} \times \mathbb{R}^{d}$ is ergodic and non-squashable. The same demonstration works, when taking $\left(n^{2} q_{\theta(d n+j)}^{p}\right)$ as rigid sequences.

- of class $\mathcal{C}^{\infty}$, if $\lim \sup q_{n+1} / q_{n}^{p}=+\infty$, for all $p \geq 1$. Choose this time $(\theta(k))$ satisfying $q_{\theta(k)+1} \geq$ $k^{3} q_{\theta(k)}^{k}$ and introduce :

$$
f=\left(f^{i}\right)_{1 \leq i \leq d}, \text { with } f^{i}(x)=\sum_{k \geq 1} k^{-2} q_{\theta(d k+i)}^{-k} \sin \left(2 \pi q_{\theta(d k+i)} x\right)
$$

Then $T_{f}: \mathbb{T}^{1} \times \mathbb{R}^{d} \rightarrow \mathbb{T}^{1} \times \mathbb{R}^{d}$ is ergodic and non-squashable. Take $\left(n^{2} q_{\theta(d n+j)}^{n}\right)$ as rigid sequences.

- real analytic, if $\lim \sup \log q_{n+1} / q_{n}=+\infty$. Choose $(\theta(n))$ checking $q_{\theta(n)+1} /\left(q_{\theta(n)} e^{\left.n q_{\theta(n)}\right)} \rightarrow\right.$ $+\infty$ and define :

$$
f=\left(f^{i}\right)_{1 \leq i \leq d}, \text { with } f^{i}(x)=\sum_{k \geq 1} \kappa_{k, i}^{-1} \sin \left(2 \pi q_{\theta(d k+i)} x\right),
$$

where $\kappa_{k, i}=q_{\theta(d k+i)}\left[\exp \left\{(d k+i) q_{\theta(d k+i)}\right\}\right]$ and $[x]$ is the integer part of $x$. Each $f^{i}$ is real analytic, since $z \longmapsto \sum_{k \geq 1} \kappa_{k, i}^{-1} \exp \left(z q_{\theta(d k+i)}\right)$ uniformly converges on compact sets of $\mathbb{C}$ and $T_{f}: \mathbb{T}^{1} \times \mathbb{R}^{d} \rightarrow \mathbb{T}^{1} \times \mathbb{R}^{d}$ is ergodic and non-squashable, using now $\left(\kappa_{n, j}\right)$ as rigid sequences.

Remark 3 - Fixing $d=1$, we recall a few facts about coboundaries. If $\alpha$ has bpq and $f^{\prime} \in L^{2}\left(\mathbb{T}^{1}\right)$, then $f$ is a $L^{2}$-coboundary, in particular when $f$ is $\mathcal{C}^{1}$. If $\alpha$ has Diophantine type 1 and $f$ is $\mathcal{C}^{1+\varepsilon}$, then $f$ is a continuous coboundary (cf Arnold [3]). A result of Meyer (cf Herman [11], p. 187) says that for any irrational $\alpha$ there is $f$ of class $\mathcal{C}^{1}$ that is not a continuous coboundary.

\subsection{Adequate perturbations for some $B V$ functions}

Consider the same context with $d=1$ and any irrational $\alpha$. The function :

$$
h(x)=\sum_{l \geq 1} q_{l^{2}}^{-1} \sin \left(2 \pi q_{l^{2}} x\right)
$$

is an example such that $\left(h_{n}\right)$ converges in law along a subsequence of the $\left(q_{n}\right)$ to a random variable whose support contains a non-empty open interval. Such a map can be used as a "good" perturbing element for a large class of BV functions.

Proposition 2.5.

Consider $\left(a_{k}\right)_{k \geq 1} \in l^{1}(\mathbb{N})$ and intervals $\left(I_{k}\right)_{k \geq 1}$ in $\mathbb{T}^{1}$. Set $\gamma=\sum_{k \geq 1} a_{k} \mu\left(I_{k}\right)$ and define :

$$
f=\sum_{k \geq 1} a_{k} 1_{I_{k}}-\gamma
$$

Introduce the compact $K=\left\{\sum a_{k} u_{k} \mid u_{k} \in \mathbb{Z} \cap[-2,2]\right\}$. We have :

- If $K$ has empty interior, then $T_{f+\varepsilon h}: \mathbb{T}^{1} \times \mathbb{R} \rightarrow \mathbb{T}^{1} \times \mathbb{R}$ is ergodic for any $\varepsilon \neq 0$.

- If the box-dimension of $K$ is $<1 / 2$, then $T_{f+\varepsilon h}$ is ergodic and non-squashable for any $\varepsilon \neq 0$.

Proof of the proposition :

Since $\operatorname{Var}\left(1_{I_{k}}\right)=2$, the Denjoy-Koksma inequality gives :

$$
\left(1_{I_{k}}-\mu\left(I_{k}\right)\right)_{q_{n}}(x)=m_{k, n}(x)-\left\{q_{n} \mu\left(I_{k}\right)\right\} \in[-2,2], \text { where } m_{k, n}(x) \in \mathbb{Z} \cap[-2,2] .
$$


As in the proof of proposition $2.4 \mathrm{ii}$ ), we have :

$$
h_{q_{n^{2}}}(x)=\chi_{n} \sin \left[q_{n^{2}} \pi\left(2 x+\left(q_{n^{2}}-1\right) \alpha\right)\right]+o(1),
$$

with uniform $o(1)$ and where the $\left(\chi_{n}\right)$ are uniformly far from 0 and $\pm \infty$. Choose $(\theta(n))_{n \geq 1}$ so that $\gamma_{n}:=\sum_{k \geq 1} a_{k}\left\{q_{\theta(n)^{2}} \mu\left(I_{k}\right)\right\} \rightarrow \gamma_{\infty}$ and $\chi_{\theta(n)} \rightarrow \chi \neq 0$. Introduce the random variables :

$$
X_{n}(x)=\varepsilon h_{q_{\theta(n)^{2}}}(x), Y_{n}(x)=\sum_{k \geq 1} a_{k} m_{k, \theta(n)^{2}}(x) \text { and } Z_{n}=X_{n}+Y_{n}
$$

Let $X$ be the uniform random variable $x \longmapsto x$ on $\left(\mathbb{T}^{1}, \mu\right)$. We have :

$$
X_{n} \rightarrow \varepsilon \chi \sin (2 \pi X), \text { in law. }
$$

Since the $\left(X_{n}\right)$ and the $\left(Y_{n}\right)$ are uniformly bounded, the laws of the $\left(Z_{n}\right)$ are tight. Let $\left(r_{n}\right)$ be a sequence such that $\left(Z_{r_{n}}\right)$ converges in law to some $Z$. Since $\gamma_{n} \rightarrow \gamma_{\infty}$, notice that $\left(Z_{r_{n}}, \gamma_{r_{n}}\right)$ converges in law to $\left(Z, \gamma_{\infty}\right)$.

Let $\varepsilon \neq 0$ and suppose that $T_{f+\varepsilon h}$ is not ergodic. Then $\mathcal{E}(f+\varepsilon h)=\lambda \mathbb{Z}$, for some $\lambda \geq 0$. Fixing $\delta>0$, introduce the open set $O_{\delta}=\left\{x \in \mathbb{T}^{1}, \operatorname{dist}(x, \lambda \mathbb{Z})<\delta\right\}$ and its closure $F_{\delta}$. By lemma 2.2 :

$$
1=\mu\left(Z-\gamma_{\infty} \in O_{\delta}\right)
$$

Since $O_{\delta}+\gamma_{\infty}$ is open, using the law convergence for the first inequality and the fact that $Y_{r_{n}} \in K$, as well as $K=-K$, for the last inequality, we obtain :

$$
\begin{aligned}
1 \leq \liminf \mu\left(Z_{r_{n}} \in O_{\delta}+\gamma_{\infty}\right) & \leq \limsup \mu\left(Z_{r_{n}} \in F_{\delta}+\gamma_{\infty}\right) \\
& \leq \limsup \mu\left(X_{r_{n}} \in F_{\delta}+K+\gamma_{\infty}\right) .
\end{aligned}
$$

Since $F_{\delta}+K+\gamma_{\infty}$ is closed, as $K$ is compact, using the law convergence of $\left(X_{n}\right)$ we finally get :

$$
1=\mu\left(\varepsilon \chi \sin (2 \pi X) \in F_{\delta}+K+\gamma_{\infty}\right)
$$

The closedness of $F_{\delta}+K+\gamma_{\infty}$ then implies that $\varepsilon \chi[-1,1] \subset \gamma_{\infty}+K+F_{\delta}$. As $K$ is compact, $K+F_{\delta}$ tends to $K+\lambda \mathbb{Z}$ as $\delta \rightarrow 0$. Therefore :

$$
\varepsilon \chi[-1,1] \subset \gamma_{\infty}+\cup_{n \in \mathbb{Z}}(K+n \lambda) .
$$

However each $K+n \lambda$ is a compact with empty interior, thus $\cup_{n \in \mathbb{Z}}(K+n \lambda)$ also has empty interior, but this is a contradiction. Therefore $T_{f+\varepsilon h}$ is ergodic.

Suppose now that $\operatorname{dim}_{b o x}(K)<1 / 2$ and $\varepsilon \neq 0$. If $T_{f+\varepsilon h}$ is squashable, by proposition 2.3 there exists $\kappa \neq \pm 1$, a measurable $\psi: \mathbb{T}^{1} \rightarrow \mathbb{R}^{d}$ and $\beta$ such that for almost-every $x \in \mathbb{T}^{1}$ :

$$
g(x):=\kappa(f+\varepsilon h)(x)-(f+\varepsilon h)(x+\beta)=\psi(x)-\psi(x+\alpha) .
$$

Set $\tilde{f}(x)=\kappa f(x)-f(x+\beta)=\kappa \sum a_{k} 1_{I_{k}}(x)-\sum a_{k} 1_{I_{k}-\beta}(x)-\gamma(\kappa-1)$. Comparing with $f$, the compact $K$ is replaced in $\tilde{f}$ by the compact $\kappa K+K$, whose Hausdorff dimension is less than $2 \operatorname{dim}_{\text {box }}(K)<1$. Thus $\kappa K+K$ has empty interior.

Choose $\left(k_{n}\right)$ so that $q_{\theta\left(k_{n}\right)^{2}} \beta \rightarrow \zeta \bmod (1)$. If $T_{g}$ is not ergodic, then the same proof with $\tilde{f}$ and the perturbation $\varepsilon(\kappa h()-.h(.+\beta))$, using the sequence $\left(q_{\theta\left(k_{n}\right)^{2}}\right)$, gives some $\lambda \geq 0$ so that :

$$
\varepsilon \chi(\kappa \sin (2 \pi c)-\sin (2 \pi(c+\zeta))) \in \gamma_{\infty}(\kappa-1)+\cup_{n \in \mathbb{Z}}(\kappa K+K+n \lambda), \forall c \in[0,1] .
$$


However, the image of the left-hand side contains a non-empty open interval, since $|\kappa| \neq 1$, whereas the right-hand side has empty interior. Therefore $T_{g}$ is ergodic. This contradicts (2.5) and concludes the proof of the proposition.

\subsection{An example with $\varphi: \mathbb{T}^{2} \rightarrow \mathbb{R}^{2}$}

Take now $X=\mathbb{T}^{2}$ with Lebesgue measure $\mu$ and $G=\mathbb{R}^{2}$. The method used in the proof of proposition 2.4 furnishes for any minimal translation $T$ by $\alpha=\left(\alpha_{1}, \alpha_{2}\right)$ on $\mathbb{T}^{2}$ a continuous $f$ : $\mathbb{T}^{2} \rightarrow \mathbb{R}^{2}$ of the form $f(x, y)=(\varphi(x), \varphi(y))$ such that $T_{f}: \mathbb{T}^{2} \times \mathbb{R}^{2} \rightarrow \mathbb{T}^{2} \times \mathbb{R}^{2}$ is ergodic and nonsquashable. Indeed, it is enough to take a sequence $\left(q_{n}\right)$ growing fast enough, with $\left(\left(q_{n} \alpha_{1}\right)\right)+$ $\left.\left(\left(q_{n} \alpha_{2}\right)\right)\right) \rightarrow 0$ fast enough. Setting :

$$
\varphi(x)=\sum_{k \geq 1} q_{2 k}^{-1} \sin \left(2 \pi q_{2 k+1} x\right)
$$

the same proof works with the rigid sequence $\left(q_{2 n}\right)$.

Inspired by ideas of Yoccoz [21] (see also Conze-Chevallier [5]), we build a smooth example for a particular $\alpha$.

Proposition 2.6.

Let $\alpha=\left(\alpha_{1}, \alpha_{2}\right)=\left(\sum_{k \geq 1} 2^{-m_{k}}, \sum_{k \geq 1} 3^{-m_{k}}\right)$, where $m_{n+1} / m_{n}>\ln 12 / \ln 2$. Define :

$$
f(x, y)=(\varphi(x), \varphi(y)), \text { where } \varphi(x)=\sum_{k \geq 1} k^{-2} 6^{-m_{k}} \sin \left(2 \pi 6^{m_{k}} x\right) .
$$

Then the translation $T$ by $\alpha$ is minimal, $f$ is of class $\mathcal{C}^{1}$ and $T_{f}: \mathbb{T}^{2} \times \mathbb{R}^{2} \rightarrow \mathbb{T}^{2} \times \mathbb{R}^{2}$ is ergodic and non-squashable.

Proof of the proposition :

We first check that $T$ is minimal. Considering $\alpha^{n}=\sum_{k \leq n}\left(2^{-m_{k}}, 3^{-m_{k}}\right)$, the subgroup generated by $\alpha^{n}$ is $\left\langle\alpha^{n}\right\rangle=\left(k 2^{-m_{n}}, l 3^{-m_{n}}\right)_{(k, l) \in \mathbb{Z}^{2}}$ and $\alpha^{n}$ has order $6^{m_{n}}$. For $0 \leq q \leq 6^{m_{n}}$, we have :

$$
d\left(q \alpha, q \alpha^{n}\right) \leq C 6^{m_{n}} 2^{-m_{n+1}} \rightarrow 0
$$

so the orbit of $\alpha$ is dense in $\mathbb{T}^{2}$.

Set $t_{n}=n^{2} 6^{m_{n}}$. Since $\left(\left(t_{n} \alpha_{i}\right)\right) \leq n^{2} 6^{m_{n}} 2^{-m_{n+1}} \rightarrow 0,\left(t_{n}\right)$ is a rigid sequence for $\alpha=\left(\alpha_{1}, \alpha_{2}\right)$. Let $q_{n}=6^{m_{n}}$ and consider $\varphi_{n}$ under the rotation by $\alpha_{i}$. Using the decomposition given in (2.3) :

$$
\left|A_{n}\right| \leq C \sum_{k<n} \frac{n^{2}\left(\left(q_{n} \alpha_{i}\right)\right)}{k^{2}\left(\left(q_{k} \alpha_{i}\right)\right)} \leq C \frac{n^{2} 6^{m_{n}} 2^{-m_{n+1}}}{6^{m_{n-1}} 2^{-m_{n}}} \rightarrow 0 \text { and }\left|C_{n}\right| \leq C \frac{q_{n}}{q_{n+1}} \rightarrow 0
$$

As $\sin \left(\pi 6^{m_{n}} n^{2} 6^{m_{n}} \alpha_{i}\right) / n^{2} 6^{m_{n}} \sin \left(\pi 6^{m_{n}} \alpha_{i}\right) \rightarrow 1$, we obtain :

$$
f_{t_{n}}(x, y)=\left(\sin \left[\pi q_{n}\left(2 x+\left(t_{n}-1\right) \alpha_{1}\right)\right], \sin \left(\left[\pi q_{n}\left(2 y+\left(t_{n}-1\right) \alpha_{2}\right)\right]\right)+o(1) .\right.
$$

Denoting by $\mathcal{L}$ the law of $\sin (2 \pi X)$, where $X$ is a uniform random variable on $[0,1]$, we have $f_{t_{n}} \rightarrow \mathcal{L} \otimes \mathcal{L}$, in law. Lemma 2.2 gives $[-1,1]^{2} \subset \mathcal{E}(f)$. Thus $\mathcal{E}(f)=\mathbb{R}^{2}$ and $T_{f}$ is ergodic.

If $T_{f}$ is squashable, proposition 2.3 furnishes a $2 \times 2$ invertible matrix $M$ with $|\operatorname{det} M| \neq 1$, a measurable $\psi: \mathbb{T}^{2} \rightarrow \mathbb{R}^{2}$ and $\beta=\left(\beta_{1}, \beta_{2}\right)$ such that for almost-every $x \in \mathbb{T}^{2}:$

$$
g(x):=M f(x)-f(x+\beta)=\psi(x)-\psi(x+\alpha)
$$


We thus have $\mathcal{E}(g)=\{0\}$. Consider a subsequence $\left(k_{n}\right)$ so that $q_{k_{n}} \beta \rightarrow \zeta=\left(\zeta_{1}, \zeta_{2}\right) \bmod \mathbb{Z}^{2}$. Using the sequence $\left(t_{k_{n}}\right)$, we obtain via the same proof that for all $(x, y) \in \mathbb{R}^{2}$ :

$$
M\left(\begin{array}{l}
\sin (2 \pi x) \\
\sin (2 \pi y)
\end{array}\right)-\left(\begin{array}{c}
\sin \left(2 \pi\left(x+\zeta_{1}\right)\right) \\
\sin \left(2 \pi\left(y+\zeta_{2}\right)\right)
\end{array}\right)=0 .
$$

Taking $y=0$, we get $\sin (2 \pi x) M e_{1}={ }^{t}\left(\sin \left(2 \pi\left(x+\zeta_{1}\right)\right), \sin \left(2 \pi \zeta_{2}\right)\right)$. Thus $M_{21}=0$ and this gives $M_{11} \sin (2 \pi x)=\sin \left(2 \pi\left(x+\zeta_{1}\right)\right)$. Necessarily $M_{11}= \pm 1$. In the same way, $M_{12}=0$ and $M_{22}= \pm 1$. As a consequence $M$ is diagonal and $\operatorname{det} M= \pm 1$, but this is a contradiction.

\section{Smooth ergodic cocycles in a nilpotent group}

We come back to $X=\mathbb{T}^{1}$, with an irrational rotation $T$ of angle $\alpha$ and Lebesgue measure $\mu$, and use the same notations as in section 2.3 for the sequence of best approximations of $\alpha$. We now build examples of smooth ergodic cocycles with values in the group $G=N_{d}(\mathbb{R})$, where :

$$
N_{d}(\mathbb{R})=\left\{\left(\begin{array}{cccc}
1 & & \cdots & \\
0 & \ddots & \left(a^{i j}\right) & \vdots \\
\vdots & \ddots & \ddots & \\
0 & \cdots & 0 & 1
\end{array}\right) \mid\left(a^{i j}\right)=\left(a^{i j}\right)_{1 \leq i<j \leq d} \in \mathbb{R}^{d(d-1) / 2}\right\}
$$

If $A \in N_{d}(\mathbb{R})$, we write $A=\left(a^{i j}\right)_{1 \leq i<j \leq d}$ or $A=\left(a^{i j}\right)$ for simplicity. Matricial indices are written upwards, down indices being for cocycles.

We suppose that $d \geq 3$, so $N_{d}(\mathbb{R})$ is nilpotent but non-abelian. Recall that Haar measure in $N_{d}(\mathbb{R})$ is the left and right-invariant Lebesgue measure.

THEOREM 3.1.

Let $\alpha$ have non bpq. Choose a strictly increasing $(\theta(n))_{n \geq 1}$ satisfying $a_{\theta(n)+1} \geq n^{3}$ as well as $q_{\theta(n+1)} \geq q_{\theta(n)+1}^{d}$. We define :

$$
(\Phi)=\left(f^{i j}\right)_{1 \leq i<j \leq d}, \text { by } f^{i j}(x)=\sum_{k \geq 1} \frac{\sin \left(2 \pi q_{\theta\left(d^{2} k+d i+j\right)} x\right)}{k^{2} q_{\theta\left(d^{2} k+d i+j\right)}}
$$

Then $\Phi$ is $\mathcal{C}^{1}$ and $T_{\Phi}: \mathbb{T}^{1} \times N_{d}(\mathbb{R}) \rightarrow \mathbb{T}^{1} \times N_{d}(\mathbb{R})$ is ergodic.

Remark 1 - For almost-every $x \in \mathbb{T}^{1}$, the orbit $\left(\Phi_{n}(x)\right)_{n \in \mathbb{Z}}\left(\right.$ and in fact $\left.\left(\Phi_{n}(x)\right)_{n \geq 1}\right)$ is dense in $N_{d}(\mathbb{R})$. The continuity of $\Phi$ implies that this property is true for $x$ in a $G_{\delta}$-set of full Lebesgue measure.

Remark 2 - If $\alpha$ has bpq and $\Phi$ is $\mathcal{C}^{1}$ or if $\alpha$ has Diophantine type 1 and $\Phi$ is $\mathcal{C}^{1+\varepsilon}$, then $T_{\Phi}$ cannot be ergodic. Indeed $\Phi_{n}^{12}=f_{n}^{12}$, for all $n \in \mathbb{Z}$ and $T_{f^{12}}: \mathbb{T}^{1} \times \mathbb{R} \rightarrow \mathbb{T}^{1} \times \mathbb{R}$ would be ergodic. However $f^{12}$ is in this case a coboundary.

Remark 3 - There is a similar statement for $\alpha$ with bpq. Fixing a decreasing $\varepsilon(h) \rightarrow+\infty$, as $h \rightarrow 0^{+}$, choose $(\theta(n))_{n \geq 1}$ with $\theta(n) \geq n^{2}, \varepsilon\left(1 / q_{\theta(n)}\right) \geq n$ and $q_{\theta(n+1)} \geq q_{\theta(n)+1}^{d}$. Set :

$$
(\Phi)=\left(f^{i j}\right)_{1 \leq i<j \leq d}, \text { where } f^{i j}(x)=\sum_{k \geq 1} q_{\theta\left(d^{2} k+d i+j\right)}^{-1} \sin \left(2 \pi q_{\theta\left(d^{2} k+d i+j\right)} x\right) .
$$

Then $h \varepsilon(h)$ is a modulus of continuity for $\Phi$ and $T_{\Phi}: \mathbb{T}^{1} \times N_{d}(\mathbb{R}) \rightarrow \mathbb{T}^{1} \times N_{d}(\mathbb{R})$ is ergodic. The proof is directly adapted from the one given below, as for item $i i$ ) of proposition 2.4 from item $i$ ).

Remark 4 - We examine squashability in the special case when $d=3$ below. 
As $N_{d}(\mathbb{R})$ is not abelian, lemma 2.2 does not apply. We proceed differently and first introduce some definitions. Denote by $D_{l}(A)=\left(a^{i i+l}\right)_{1 \leq i \leq d-l}$ the $l$-upper diagonal of $A=\left(a^{i j}\right) \in N_{d}(\mathbb{R})$.

DEFINITION 3.2 .

A l-upper diagonal $x=\left(x^{i i+l}\right)_{1 \leq i \leq d-l} \in \mathbb{R}^{d-l}$ is essential for $\Phi$ if $\forall \varepsilon>0, \forall$ Borel set $B$ with $\mu(B)>$ 0 , there is $n \in \mathbb{Z}$ such that :

$$
\mu\left(B \cap T^{-n} B \cap\left\{\left(D_{1}\left(\Phi_{n}\right), \cdots, D_{l}\left(\Phi_{n}\right)\right) \in V_{\varepsilon}\left(0_{\mathbb{R}^{d-1}}, \cdots, 0_{\mathbb{R}^{d-l+1}}, x\right)\right\}\right)>0 .
$$

We write $\mathcal{D}_{l}(\Phi)$ for the set of l-upper essential diagonals.

A generalisation of considerations by Greschonig [10] is the following.

LEMMA 3.3 .

The set $\mathcal{D}_{l}(\Phi)$ is a closed subgroup of $\left(\mathbb{R}^{d-l},+\right)$ and :

$$
\mathcal{E}(\Phi)=N_{d}(\mathbb{R}) \Leftrightarrow \mathcal{D}_{l}(\Phi)=\mathbb{R}^{d-l}, \text { for } 1 \leq l \leq d-1
$$

Proof of the lemma :

We first show that $\mathcal{D}_{l}(\Phi)$ is a closed subgroup of $\left(\mathbb{R}^{d-l},+\right)$. Let $x$ and $x^{\prime}$ be in $\mathcal{D}_{l}(\Phi)$. Fix $\varepsilon>0$ and a Borel set $B$ with $\mu(B)>0$. For some $m \in \mathbb{Z}$ :

$$
\mu\left(B \cap T^{-m} B \cap\left\{\left(D_{1}\left(\Phi_{m}\right), \cdots, D_{l}\left(\Phi_{m}\right)\right) \in V_{\varepsilon}\left(0_{\mathbb{R}^{d-1}}, \cdots, 0_{\mathbb{R}^{d-l+1}}, x\right)\right\}\right)>0 .
$$

Let $C$ be the set appearing above. Since $\mu\left(T^{m} C\right)=\mu(C)>0$, there is $n \in \mathbb{Z}$ such that :

$$
\mu\left(T^{m} C \cap T^{m-n} C \cap\left\{\left(D_{1}\left(\Phi_{n}\right), \cdots, D_{l}\left(\Phi_{n}\right)\right) \in V_{\varepsilon}\left(0_{\mathbb{R}^{d-1}}, \cdots, 0_{\mathbb{R}^{d-l+1}}, x^{\prime}\right)\right\}\right)>0 .
$$

The following fact can be verified by carefully considering the entries of the matrix product : if $P$ and $Q$ in $N_{d}(\mathbb{R})$ are such that :

$$
\left(D_{j}(Q)\right)_{1 \leq j \leq l} \in V_{\varepsilon}\left(\left(0_{\mathbb{R}^{d-j}}\right)_{1 \leq j \leq l-1}, x^{\prime}\right) \text { and }\left(D_{j}(P)\right)_{1 \leq j \leq l} \in V_{\varepsilon}\left(\left(0_{\mathbb{R}^{d-j}}\right)_{1 \leq j \leq l-1}, x\right),
$$

then $\left(D_{j}(Q P)\right)_{1 \leq j \leq l} \in V_{M \varepsilon}\left(\left(0_{\mathbb{R}^{d-j}}\right)_{1 \leq j \leq l-1}, x+x^{\prime}\right)$, where $M$ is a constant depending only on $d$. If $F$ is the set in (3.1), using the cocycle property :

$$
T^{-m} F \subset B \cap T^{-m-n} B \cap\left\{\left(D_{1}\left(\Phi_{m+n}\right), \cdots, D_{l}\left(\Phi_{m+n}\right)\right) \in V_{M \varepsilon}\left(0_{\mathbb{R}^{d-1}}, \cdots, 0_{\mathbb{R}^{d-l+1}}, x+x^{\prime}\right)\right\} .
$$

Since $\mu\left(T^{-m} F\right)=\mu(F)>0$, we conclude that $x+x^{\prime} \in \mathcal{D}_{l}(\Phi)$. Similarly, $-x \in \mathcal{D}_{l}(\Phi)$, if $x \in \mathcal{D}_{l}(\Phi)$. Hence $\mathcal{D}_{l}(\Phi)$ is a subgroup of $\left(\mathbb{R}^{d-l},+\right)$. The closedness part is obvious.

We turn to the second part of the lemma. Clearly, $\mathcal{E}(\Phi)=N_{d}(\mathbb{R})$ implies that $\mathcal{D}_{l}(\Phi)=\mathbb{R}^{d-l}$, for all $1 \leq l \leq d-1$. Conversely, fix $a=\left(a^{i j}\right) \in N_{d}(\mathbb{R}), \varepsilon>0$ and a Borel set $B$ with $\mu(B)>0$.

Since $a^{1}:=\left(a^{i i+1}\right)_{1 \leq i \leq d-1} \in \mathcal{D}_{1}(\Phi)$, there is $n_{1}$ so that $\mu\left(B \cap T^{-n_{1}} B \cap\left\{D_{1}\left(\Phi_{n_{1}}\right) \in V_{\varepsilon}\left(a^{1}\right)\right\}\right)>0$. Let now $\left(b_{l}\right)_{l \geq 1}$ be dense in $N_{d}(\mathbb{R})$. As :

$$
\left\{D_{1}\left(\Phi_{n_{1}}\right) \in V_{\varepsilon}\left(a^{1}\right)\right\}=\cup_{l \geq 1}\left\{\Phi_{n_{1}} \in V_{\varepsilon}\left(b_{l}\right), D_{1}\left(\Phi_{n_{1}}\right) \in V_{\varepsilon}\left(a^{1}\right)\right\},
$$

there is $b^{1}=\left(b^{i j, 1}\right)_{1 \leq i<j \leq d} \in N_{d}(\mathbb{R})$ such that $b^{i i+1,1}=a^{i i+1}$, for all $1 \leq i \leq d-1$, so that :

$$
\mu\left(B \cap T^{-n_{1}} B \cap\left\{\Phi_{n_{1}} \in V_{\varepsilon}\left(b^{1}\right)\right\}\right)>0 .
$$

Let $B_{2}$ be the set appearing above. Since $a^{2}:=\left(a^{i i+2}-b^{i i+2,1}\right)_{1 \leq i \leq d-2} \in \mathcal{D}_{2}(\Phi)$ and $\mu\left(T^{n_{1}} B_{2}\right)>0$, there is $n_{2}$ such that : 


$$
\mu\left(T^{n_{1}} B_{2} \cap T^{n_{1}-n_{2}} B_{2} \cap\left\{\left(D_{1}\left(\Phi_{n_{2}}\right), D_{2}\left(\Phi_{n_{2}}\right)\right) \in V_{\varepsilon}\left(0_{\mathbb{R}^{d-1}}, a^{2}\right)\right\}\right)>0 .
$$

If $B_{3}$ is the previous set, then $\mu\left(T^{-n_{1}} B_{3}\right)=\mu\left(B_{3}\right)>0$ and :

$$
T^{-n_{1}} B_{3} \subset B \cap T^{-n_{1}-n_{2}} B \cap\left\{\left(D_{1}\left(\Phi_{n_{1}+n_{2}}\right), D_{2}\left(\Phi_{n_{1}+n_{2}}\right)\right) \in V_{M \varepsilon}\left(\left(a^{i i+1}\right)_{1 \leq i \leq d-1},\left(a^{i i+2}\right)_{1 \leq i \leq d-2}\right)\right\},
$$

where $M$ is as in (3.2). The separability of $N_{d}(\mathbb{R})$ again implies that there is $b^{2}=\left(b^{i j, 2}\right)_{1 \leq i<j \leq d} \in$ $N_{d}(\mathbb{R})$, with $b^{i i+l, 2}=a^{i i+l}$ for $l \in\{1,2\}$ and $1 \leq i \leq d-l$, such that :

$$
\mu\left(B \cap T^{-n_{1}-n_{2}} B \cap\left\{\Phi_{n_{1}+n_{2}} \in V_{2 M \varepsilon}\left(b^{2}\right)\right\}\right)>0 .
$$

Iterating the procedure, we obtain integers $n_{1}, \cdots, n_{d-1}$ such that :

$$
\mu\left(B \cap T^{-n_{1}-n_{2}-\cdots-n_{d-1}} B \cap\left\{\Phi_{n_{1}+n_{2}+\cdots+n_{d-1}} \in V_{(2 M)^{d-2} \varepsilon}(a)\right\}\right)>0 .
$$

As a result, $a=\left(a^{i j}\right) \in \mathcal{E}(\Phi)$. Thus $\mathcal{E}(\Phi)=N_{d}(\mathbb{R})$ and this concludes the proof of the lemma.

Proof of theorem 3.1:

Step 1. We make preliminary computations. Recall that $\Phi_{n}=T^{n-1} \Phi \cdots \Phi$, for $n \geq 1$. We have $\Phi_{n}^{k k}=1$ and by definition of the matrix product, if $k<l$ :

$$
\Phi_{n}^{k l}=\sum_{m=1}^{l-k} \sum_{k=u_{0}<\cdots<u_{m}=l} \sum_{0 \leq s_{m}<\cdots<s_{1}<n} T^{s_{1}} f^{u_{0} u_{1}} \cdots T^{s_{m}} f^{u_{m-1} u_{m}} .
$$

Using the decomposition $\sin \left(2 \pi q_{\theta\left(d^{2} r+d k+l\right)} x\right)=\left(e^{2 i \pi q_{\theta\left(d^{2} r+d k+l\right)} x}-e^{-2 i \pi q_{\theta\left(d^{2} r+d k+l\right)} x}\right) / 2 i$, write $f^{k l}=\left(f^{k l,+}-f^{k l,-}\right) / 2 i$. Then :

$$
\Phi_{n}^{k l}=\sum_{m=1}^{l-k} \frac{1}{(2 i)^{m}} \sum_{\delta=\left(\delta_{1}, \cdots, \delta_{m}\right) \in\{ \pm 1\}} \delta_{1} \cdots \delta_{m} \sum_{k=u_{0}<\cdots<u_{m}=l} \Phi_{n}^{k l, m, \delta, u},
$$

where $u=\left(u_{i}\right)$ and :

$$
\Phi_{n}^{k l, m, \delta, u}=\sum_{0 \leq s_{m}<\cdots<s_{1}<n} T^{s_{1}} f^{u_{0} u_{1}, \delta_{1}} \cdots T^{s_{m}} f^{u_{m-1} u_{m}, \delta_{m}}
$$

Set $w_{r}(k, l)=d^{2} r+d k+l$ and $v_{t}(p)=q_{\theta\left(w_{p}\left(u_{t-1}, u_{t}\right)\right)}$. Using the expression for $f^{k l, \pm}$, we obtain :

$$
\Phi_{n}^{k l, m, \delta, u}(x)=\sum_{p_{1}, \cdots, p_{m} \geq 1} \frac{e^{2 i \pi \sum_{t=1}^{m} \delta_{t} v_{t}\left(p_{t}\right) x}}{\prod_{t=1}^{m} p_{t}^{2} v_{t}\left(p_{t}\right)} \sum_{0 \leq s_{m}<\cdots<s_{1}<n} e^{2 i \pi \delta_{1} v_{1}\left(p_{1}\right) s_{1} \alpha} \cdots e^{2 i \pi \delta_{m} v_{m}\left(p_{m}\right) s_{m} \alpha} .
$$

Step 2. A remark, about indices, is that $w_{r}(k, l)=w_{r^{\prime}}\left(k^{\prime}, l^{\prime}\right)$ if and only if $r=r^{\prime}, k=k^{\prime}$ and $l=l^{\prime}$, since $d k+l \in\left[1, d^{2}\right]$ and $l \in[1, d]$. Therefore, for all values of $p_{1}, \cdots, p_{m}$, the quantities $\left(w_{p_{t}}\left(u_{t-1}, u_{t}\right)\right)_{1 \leq t \leq m}$ are pairwise distinct.

Fix $(i, j)$ with $1 \leq i<j \leq d$ and suppose that $(k, l)$ satisfies $l-k \leq j-i$. In other words $(k, l)$ lies on a lower upper-diagonal than $(i, j)$. Impose also $(k, l) \neq(i, j)$. As a result, for all $m, \delta, u$ and for all $p_{1}, \cdots, p_{m}$, the $m$-tuples $\left(w_{p_{t}}\left(u_{t-1}, u_{t}\right)\right)_{1 \leq t \leq m}$ appearing in the expression of $\Phi_{n}^{k l, m, \delta, u}$ are also distinct from any $w_{n}(i, j), n \geq 1$.

Let now $t_{n}=n^{2} q_{\theta\left(w_{n}(i, j)\right)}$. We prove that $\Phi_{t_{n}}^{k l, m, \delta, u} \rightarrow 0$ uniformly, as $n \rightarrow+\infty$. Let $I_{n}$ be the set of $\left(p_{1}, \cdots, p_{m}\right)$ so that $\max _{1 \leq t \leq m} w_{p_{t}}\left(u_{t-1}, u_{t}\right)<w_{n}(i, j)$. Then in (3.3) at time $t_{n}$, the sum restricted to $I_{n}^{c}$ and written $S_{1}$ satisfies, for some constant $C>0$ : 


$$
\begin{aligned}
\left|S_{1}\right| & \leq \sum_{t=1}^{m} \sum_{p_{1}, \cdots, p_{m} \geq 1 ; w_{p_{t}}\left(u_{t-1}, u_{t}\right)>w_{n}(i, j)} \frac{t_{n}^{m}}{\prod_{t^{\prime}=1}^{m} p_{t^{\prime}}^{2} v_{t^{\prime}}\left(p_{t^{\prime}}\right)} \\
& \leq t_{n}^{m} \sum_{t=1}^{m} \prod_{t_{t^{\prime} \in\{1, \cdots, m\} \backslash\{t\}}}\left(\sum_{p_{t^{\prime}} \geq 1} \frac{1}{p_{t^{\prime}}^{2} v_{t^{\prime}}\left(p_{t^{\prime}}\right)}\right)_{p_{t} \geq 1, w_{p_{t}}\left(u_{t-1}, u_{t}\right)>w_{n}(i, j)} \frac{1}{p_{t}^{2} v_{t}\left(p_{t}\right)} \\
& \leq C n^{2 m} \frac{q_{\theta\left(w_{n}(i, j)\right)}^{m}}{q_{\theta\left(w_{n}(i, j)+1\right)}} \rightarrow_{n \rightarrow+\infty} 0 .
\end{aligned}
$$

We next turn to the sum $S_{2}$, corresponding to (3.3) at time $t_{n}$ with indices $\left(p_{1}, \cdots, p_{m}\right)$ restricted to $I_{n}$. We have :

$$
\left|S_{2}\right| \leq \sum_{p_{t} \geq 1, w_{p_{t}}\left(u_{t-1}, u_{t}\right)<w_{n}(i, j), 1 \leq t \leq m} \frac{1}{\prod_{t=1}^{m} p_{t}^{2} v_{t}\left(p_{t}\right)}\left|\Psi_{n}\left(p_{1}, \cdots, p_{m}\right)\right|,
$$

where :

$$
\begin{aligned}
\Psi_{n}\left(p_{1}, \cdots, p_{m}\right) & =\sum_{0 \leq s_{m}<\cdots<s_{1}<t_{n}} e^{2 i \pi \delta_{1} v_{1}\left(p_{1}\right) s_{1} \alpha} \cdots e^{2 i \pi \delta_{m} v_{m}\left(p_{m}\right) s_{m} \alpha} \\
& =\sum_{0 \leq s_{m-1}<\cdots<s_{1}<t_{n}} e^{2 i \pi \sum_{t=1}^{m-1} \delta_{t} v_{t}\left(p_{t}\right) s_{t} \alpha}\left(\frac{e^{2 i \pi \delta_{m} v_{m}\left(p_{m}\right) s_{m-1} \alpha}-1}{e^{2 i \pi \delta_{m} v_{m}\left(p_{m}\right) \alpha}-1}\right) \\
& =\sum_{\varepsilon_{m}=0,1}(-1)^{1+\varepsilon_{m}} \sum_{0 \leq s_{m-1}<\cdots<s_{1}<t_{n}} \frac{e^{2 i \pi\left(\sum_{t=1}^{m-1} \delta_{t} v_{t}\left(p_{t}\right) s_{t}+\varepsilon_{m} \delta_{m} v_{m}\left(p_{m}\right) s_{m-1}\right) \alpha}}{e^{2 i \pi \delta_{m} v_{m}\left(p_{m}\right) \alpha}-1} \\
= & \sum_{\varepsilon_{m}, \cdots, \varepsilon_{2}=0,1}(-1)^{m-1+\sum_{t=2}^{m} \varepsilon_{t}} \frac{e^{2 i \pi\left[\delta_{1} v_{1}\left(p_{1}\right)+\sum_{t=2}^{m} \varepsilon_{2} \cdots \varepsilon_{t} \delta_{t} v_{t}\left(p_{t}\right)\right] t_{n} \alpha}-1}{\prod_{r=1}^{m}\left(e^{2 i \pi\left[\delta_{r} v_{r}\left(p_{r}\right)+\sum_{t=r+1}^{m} \varepsilon_{r+1} \cdots \varepsilon_{t} \delta_{t} v_{t}\left(p_{t}\right)\right] \alpha}-1\right)}
\end{aligned}
$$

observing that everywhere $\delta_{r} v_{r}\left(p_{r}\right)+\sum_{t=r+1}^{m} \varepsilon_{r+1} \cdots \varepsilon_{t} \delta_{t} v_{t}\left(p_{t}\right) \neq 0$, since all $v_{t}\left(p_{t}\right)$ are pairwise distinct and, as follows easily from the hypotheses, $q_{\theta(k+1)} \geq 2 q_{\theta(k)}$ for all $k \geq 1$.

There is then an absolute constant $C>0$ such that in the last fraction, the absolute value of the denominator is bounded from below by $C \prod_{r=1}^{m}\left(\left(v_{r}\left(p_{r}\right) \alpha\right)\right)$. As a result, if $C>0$ is now generic :

$$
\begin{aligned}
\left|S_{2}\right| & \leq C \sum_{p_{t} \geq 1, w_{p_{t}}\left(u_{t-1}, u_{t}\right)<w_{n}(i, j), 1 \leq t \leq m} \frac{1}{\prod_{t=1}^{m} p_{t}^{2} v_{t}\left(p_{t}\right)} \frac{\left(\left(t_{n} \alpha\right)\right) \sum_{t=1}^{m} v_{t}\left(p_{t}\right)}{\prod_{t=1}^{m}\left(\left(v_{t}\left(p_{t}\right) \alpha\right)\right)} \\
& \leq C\left(\left(t_{n} \alpha\right)\right) \sum_{p_{t} \geq 1, w_{p_{t}}\left(u_{t-1}, u_{t}\right)<w_{n}(i, j), 1 \leq t \leq m} \frac{\prod_{t=1}^{m} q_{\theta\left(w_{p_{t}}\left(u_{t-1}, u_{t}\right)\right)+1}}{\prod_{t=1}^{m} p_{t}^{2} q_{\theta\left(w_{p_{t}}\left(u_{t-1}, u_{t}\right)\right)}} \max _{1 \leq t \leq m} q_{\theta\left(w_{p_{t}}\left(u_{t-1}, u_{t}\right)\right)} \\
& \leq C \frac{n^{2} q_{\theta\left(w_{n}(i, j)-1\right)+1} q_{\theta\left(w_{n}(i, j)-1\right)}}{q_{\theta\left(w_{n}(i, j)\right)+1}} \leq C \frac{n^{2} q_{\theta\left(w_{n}(i, j)-1\right)+1} q_{\theta\left(w_{n}(i, j)-1\right)}}{w_{n}(i, j)^{3} q_{\theta\left(w_{n}(i, j)\right)}} \\
& \leq C \frac{q_{\theta\left(w_{n}(i, j)-1\right)+1} q_{\theta\left(w_{n}(i, j)-1\right)}}{q_{\theta\left(w_{n}(i, j)-1\right)+1}^{d}} \rightarrow_{n \rightarrow+\infty} 0 .
\end{aligned}
$$

As a result, $\Phi_{t_{n}}^{k l, m, \delta, u} \rightarrow 0$, uniformly. When summing on $m, \delta$ and $u$, we get that whenever $l-k \leq j-i$ and $(k, l) \neq(i, j)$, then $\Phi_{t_{n}}^{k l} \rightarrow 0$, uniformly. Concerning $\Phi_{t_{n}}^{i j}$, write :

$$
\Phi_{t_{n}}^{i j}=f_{t_{n}}^{i j}+\sum_{m=2}^{j-i} \frac{1}{(2 i)^{m}} \sum_{\delta=\left(\delta_{1}, \cdots, \delta_{m}\right) \in\{ \pm 1\}} \delta_{1} \cdots \delta_{m} \sum_{i=u_{0}<\cdots<u_{m}=j} \Phi_{t_{n}}^{i j, m, \delta, u} .
$$

Indices $\left(u_{t-1}, u_{t}\right)$ appearing in the right-hand sum are all distinct from $(i, j)$. As before, all $\Phi_{t_{n}}^{i j, m, \delta, u}$ tend to 0 uniformly, as $n \rightarrow+\infty$. Therefore the right-hand sum tends to 0 uniformly, as $n \rightarrow+\infty$. On the contrary, proceeding as in proposition $2.4 i$ ) : 


$$
f_{t_{n}}^{i j}(x)=\kappa_{n} \sin \left(2 \pi q_{\theta\left(w_{n}(i, j)\right)} x\right)+o(1),
$$

where $\kappa_{n} \in\{ \pm 1\}$ and each $o(1)$ is uniform. Thus, $\Phi_{t_{n}}^{i j}(x)=\kappa_{n} \sin \left(2 \pi q_{\theta\left(w_{n}(i, j)\right)} x\right)+o(1)$, with uniform $o(1)$.

Step 3. We finally show that $\mathcal{E}(\Phi)=N_{d}(\mathbb{R})$. We use lemma 3.3 and prove that for all $1 \leq l \leq d-1$, we have $\mathcal{D}_{l}(\Phi)=\mathbb{R}^{d-l}$. Fix $1 \leq l \leq d-1,1 \leq i \leq d-l$. Since $\mathcal{D}_{l}(\Phi)$ is a subgroup of $\left(\mathbb{R}^{d-l},+\right)$, it is enough to show that $\{0\}^{i-1} \times[-1,1] \times\{0\}^{d-l-i} \subset \mathcal{D}_{l}(\Phi)$.

Set $j=i+l$ and $t_{n}=n^{2} q_{\theta\left(w_{n}(i, j)\right)}$. Up to considering a subsequence of the $\left(t_{n}\right)$, we suppose that $\kappa_{n} \rightarrow \kappa \in\{ \pm 1\}$, where $\kappa_{n}$ appears in (3.4). Let $c \in[0,1]$ and $x=(0, \cdots, 0, \kappa \sin (2 \pi c), 0, \cdots, 0) \in$ $\mathbb{R}^{d-l}$, where the singular component is at place $i$.

The following argument is inspired from Fayad [6]. We suppose by contradiction that $x$ is not a $l$-upper essential diagonal. Let then $\varepsilon>0$ and $B$ be a Borel set with $\mu(B)>0$ such that :

$$
\mu\left(B \cap T^{-n} B \cap\left\{\left(D_{1}\left(\Phi_{n}\right), \cdots, D_{l}\left(\Phi_{n}\right)\right) \in V_{\varepsilon}\left(0_{\mathbb{R}^{d-1}}, \cdots, 0_{\mathbb{R}^{d-l+1}}, x\right)\right\}\right)=0, \forall n \in \mathbb{Z} .
$$

Set $B_{y, \eta}=B \cap[y-\eta / 2, y+\eta / 2]$, for $\eta>0$. Since $\left(q_{\theta\left(w_{n}(i, j)\right)} y\right)_{n \geq 1} \bmod (1)$ is dense in $\mathbb{T}^{1}$ for almost-every $y$ (see for example Kuipers and Niederreiter $[\mathbf{1 2}]$ ) and $\mu(B)>0$, we can take a Lebesgue density point $y_{0} \in B$ and then a sequence $\left(k_{n}\right)=\left(k_{n}\left(y_{0}\right)\right)$ verifying the conditions :

$$
\frac{\mu\left(B_{y_{0}, \eta}\right)}{\eta} \rightarrow_{\eta \rightarrow 0} 1 \text { and } q_{\theta\left(w_{k_{n}}(i, j)\right)} y_{0} \rightarrow_{n \rightarrow+\infty} c \bmod (1) .
$$

For all $n \geq 1$ and $\eta>0$, we get from (3.5):

$$
\mu\left(B_{y_{0}, \eta} \cap T^{-t_{k_{n}}} B_{y_{0}, \eta} \cap\left\{\left(D_{1}\left(\Phi_{t_{k_{n}}}\right), \cdots, D_{l}\left(\Phi_{t_{k_{n}}}\right)\right) \in V_{\varepsilon}\left(0_{\mathbb{R}^{d-1}}, \cdots, 0_{\mathbb{R}^{d-l+1}}, x\right)\right\}\right)=0 .
$$

Choose now $\eta_{n}=n^{-1 / 2} q_{\theta\left(w_{k_{n}}(i, j)\right)}^{-1}$. Using the final result of Step 2 :

$$
\left(D_{1}\left(\Phi_{t_{k_{n}}}\right), \cdots, D_{l}\left(\Phi_{t_{k_{n}}}\right)\right) \rightarrow\left(0_{\mathbb{R}^{d-1}}, \cdots, 0_{\mathbb{R}^{d-l+1}}, x\right),
$$

uniformly on $B_{y_{0}, \eta_{n}}$. Consequently, for $n$ large enough :

$$
B_{y_{0}, \eta_{n}} \subset\left\{\left(D_{1}\left(\Phi_{t_{k_{n}}}\right), \cdots, D_{l}\left(\Phi_{t_{k_{n}}}\right)\right) \in V_{\varepsilon}\left(0_{\mathbb{R}^{d-1}}, \cdots, 0_{\mathbb{R}^{d-l+1}}, x\right)\right\} .
$$

Thus $\mu\left(B_{y_{0}, \eta_{n}} \cap T^{-t_{k_{n}}} B_{y_{0}, \eta_{n}}\right)=0$, for large $n$. Observe that $\left(\left(k_{n}^{2} q_{\theta\left(w_{k_{n}}(i, j)\right)} \alpha\right)\right) \leq 1 /\left(n q_{\theta\left(w_{k_{n}}(i, j)\right)}\right)$. Using the identity $\mu(A \cap B)=\mu(A)+\mu(B)-\mu(A \cup B)$ and the $T$-invariance of $\mu$, we obtain a contradiction in the following way :

$$
\begin{aligned}
\mu\left(B_{y_{0}, \eta_{n}} \cap T^{-t_{k_{n}}} B_{y_{0}, \eta_{n}}\right) & \geq 2 \eta_{n}(1+o(1))-\left(\eta_{n}+\left(\left(k_{n}^{2} q_{\theta\left(w_{k_{n}}(i, j)\right)} \alpha\right)\right)\right) \\
& \geq q_{\theta\left(w_{k_{n}}(i, j)\right)}^{-1}\left(\frac{1}{n^{1 / 2}}(1+o(1))-\frac{1}{n}\right)>0,
\end{aligned}
$$

for $n$ large enough. This completes the proof of the theorem.

We complete the study in the case $d=3$.

Proposition 3.4.

Let $d=3$ and $\Phi$ be as in theorem 3.1. Then $T_{\Phi}: \mathbb{T}^{1} \times N_{3}(\mathbb{R}) \rightarrow \mathbb{T}^{1} \times N_{3}(\mathbb{R})$ is also non-squashable. 
Proof of the proposition :

By proposition 2.3, if $Q: \mathbb{T}^{1} \times N_{3}(\mathbb{R}) \rightarrow \mathbb{T}^{1} \times N_{3}(\mathbb{R})$ is a non-singular transformation satisfying $Q \circ T_{\varphi}=T_{\varphi} \circ Q$, then there exist $\beta \in \mathbb{R}$, a continuous surjective group endomorphism $w: N_{3}(\mathbb{R}) \rightarrow$ $N_{3}(\mathbb{R})$ and a measurable $f: \mathbb{T}^{1} \rightarrow N_{3}(\mathbb{R})$ so that :

$$
Q(x, y)=(x+\beta, f(x) w(y)) \text { and } \Phi(x+\beta)=T f(x) w(\Phi(x)) f^{-1}(x) .
$$

If $w$ is a continuous endomorphism of $N_{3}(\mathbb{R})$, it is an exercise (see for instance Gelbrich [8], section $3)$ that there exist constants $\lambda_{1}, \gamma_{1}, \lambda_{2}, \gamma_{2}, r, s$ such that :

$$
w(a, b, c)=\left(\lambda_{1} a+\gamma_{1} b, \lambda_{2} a+\gamma_{2} b, \lambda_{1} \lambda_{2} a^{2} / 2+\gamma_{1} \gamma_{2} b^{2} / 2+\lambda_{2} \gamma_{1} a b+r a+s b+\left(\lambda_{1} \gamma_{2}-\lambda_{2} \gamma_{1}\right) c\right) .
$$

The condition that $w$ is surjective is thus equivalent to $\lambda_{1} \gamma_{2}-\lambda_{2} \gamma_{1} \neq 0$.

As we have seen in the proof of theorem 3.1, if $t_{n}=n^{2} q_{\theta\left(w_{n}(1,3)\right)}$, then there exist a sequence $\left(\kappa_{n}\right) \in\{ \pm 1\}$ and uniform $o(1)$ such that :

$$
\Phi_{t_{n}}(x)=\left(0,0, \kappa_{n} \sin \left(2 \pi q_{\theta\left(w_{n}(1,3)\right)} x\right)\right)+o(1) .
$$

Since $T^{t_{n}} x=x+t_{n} \alpha \bmod (1), t_{n} \alpha \rightarrow 0 \bmod (1)$ and $f$ is measurable, there is a deterministic subsequence $\left(u_{n}\right)$ so that $f\left(T^{t_{u_{n}}} x\right) \rightarrow f(x)$ for almost-every $x$. Suppose also that $\left(u_{n}\right)$ is chosen so that $q_{\theta\left(w_{u_{n}}(1,3)\right)} \beta$ converges to some $\zeta$ modulo one, as well as $\left(\kappa_{u_{n}}\right)$ to some $\kappa \in\{ \pm 1\}$.

Now $\left(q_{\theta\left(w_{u_{n}}(1,3)\right)} x\right)$ mod $(1)$ is dense in $[0,1)$ for almost every $x$. If $c \in[0,1)$ is fixed, then for almost-every $x$ there is a random sequence along which $\left(q_{\theta\left(w_{u_{n}}(1,3)\right)} x\right) \bmod (1)$ converges to $c$. We deduce from the relation $\Phi_{t_{n}}(x+\beta)=f\left(T^{t_{n}} x\right) w\left(\Phi_{t_{n}}(x)\right) f^{-1}(x)$ that for almost-every $x$ :

$$
(0,0, \kappa \sin (2 \pi(c+\zeta)))=f(x) w(0,0, \kappa \sin (2 \pi c)) f^{-1}(x)=w(0,0, \kappa \sin (2 \pi c)),
$$

since $w(0,0, \kappa \sin (2 \pi c))$ belongs to the center of $N_{3}(\mathbb{R})$, due to the form of $w$. The latter also implies that for all $c, \kappa \sin (2 \pi(c+\zeta))=\left(\lambda_{1} \gamma_{2}-\lambda_{2} \gamma_{1}\right) \kappa \sin (2 \pi c)$. Necessarily, $\lambda_{1} \gamma_{2}-\lambda_{2} \gamma_{1}= \pm 1$. From this property we infer that the absolute value of the Jacobian of $w$ is equal to 1 and then that $Q$ preserves the product of $\mu$ with Lebesgue measure on $N_{3}(\mathbb{R})$. As a result, $T_{\Phi}$ is not squashable.

\section{Smooth ergodic cocycles in a solvable group}

The base is still $X=\mathbb{T}^{1}$, with an irrational rotation $T$ of angle $\alpha$, Lebesgue measure $\mu$ and the notations of section 2.3. We now consider a solvable group that is not nilpotent. There are two-dimensional examples, but a classical one is $\mathrm{Sol}_{3}(\mathbb{R})$ :

$$
\operatorname{Sol}_{3}(\mathbb{R})=\left\{\left(\begin{array}{ccc}
e^{x} & 0 & y \\
0 & e^{-x} & z \\
0 & 0 & 1
\end{array}\right) \mid(x, y, z) \in \mathbb{R}^{3}\right\} .
$$

This group is the component of the identity in the planar Poincaré group and also one of "Thurston's height geometries", cf Thurston [18] or Troyanov [19]. Let us make preliminary computations.

First, $\operatorname{Sol}_{3}(\mathbb{R})$ is isomorphic to $\mathbb{R}^{3}$ with the composition law :

$$
(x, y, z) \cdot\left(x^{\prime}, y^{\prime}, z^{\prime}\right)=\left(x+x^{\prime}, e^{x} y^{\prime}+y, e^{-x} z^{\prime}+z\right) .
$$

When $A \in \operatorname{Sol}_{3}(\mathbb{R})$, we write $A=\left(A^{1}, A^{2}, A^{3}\right)$ for simplicity. Commutators are given by :

$$
(a, b, c)(x, y, z)(a, b, c)^{-1}(x, y, z)^{-1}=\left(0, b\left(1-e^{x}\right)+y\left(e^{a}-1\right), c\left(1-e^{-x}\right)+z\left(e^{-a}-1\right)\right) .
$$


The commutator subgroup $\left[\operatorname{Sol}_{3}(\mathbb{R}), \operatorname{Sol}_{3}(\mathbb{R})\right]$ is therefore $\left\{(0, u, v) \mid(u, v) \in \mathbb{R}^{2}\right\}$, which is abelian. Hence $\operatorname{Sol}_{3}(\mathbb{R})$ is solvable. Clearly $\left[\left[\operatorname{Sol}_{3}(\mathbb{R}), \operatorname{Sol}_{3}(\mathbb{R})\right], \operatorname{Sol}_{3}(\mathbb{R})\right]=\left[\operatorname{Sol}_{3}(\mathbb{R}), \operatorname{Sol}_{3}(\mathbb{R})\right]$, so $\operatorname{Sol}_{3}(\mathbb{R})$ is not nilpotent.

Mention also that in $\mathrm{Sol}_{3}(\mathbb{R})$ Haar measure is the left and right-invariant Lebesgue measure.

We next build a smooth ergodic cocycle $\left(\Phi_{n}\right)_{n \in \mathbb{Z}}$ with values in $\operatorname{Sol}_{3}(\mathbb{R})$. In comparison with $N_{d}(\mathbb{R})$, where the components of $\Phi_{n}$ were polynomial expressions, here some exponentials appear. Keeping the same strategy, we introduce for a measurable $\Phi: \mathbb{T}^{1} \rightarrow \operatorname{Sol}_{3}(\mathbb{R})$ a definition parallel to definition 3.2 .

\section{DEFINITION 4.1.}

i) A real $y$ is 2-essential for $\Phi$ if $\forall \varepsilon>0, \forall$ Borel set $B$ with $\mu(B)>0$, there is $n \in \mathbb{Z}$ such that $\mu\left(B \cap T^{-n} B \cap\left\{\Phi_{n} \in V_{\varepsilon}(0, y, 0)\right\}\right)>0$. We write $\mathcal{F}_{2}(\Phi)$ for the set of 2-essential reals.

ii) A real $z$ is 3-essential for $\Phi$ if $\forall \varepsilon>0, \forall$ Borel set $B$ with $\mu(B)>0$, there is $n \in \mathbb{Z}$ such that $\mu\left(B \cap T^{-n} B \cap\left\{\Phi_{n} \in V_{\varepsilon}(0,0, z)\right\}\right)>0$. We write $\mathcal{F}_{3}(\Phi)$ for the set of 3 -essential reals.

In the same vein as lemma 3.3, we have :

LEMMA 4.2 .

Each $\mathcal{F}_{l}(\Phi)$ is a closed subgroup of $(\mathbb{R},+)$. We have $\mathcal{E}(\Phi)=\operatorname{Sol}_{3}(\mathbb{R})$ if and only if $T_{\Phi^{1}}: \mathbb{T}^{1} \times \mathbb{R} \rightarrow$ $\mathbb{T}^{1} \times \mathbb{R}$ is ergodic and $\mathcal{F}_{2}(\Phi)=\mathcal{F}_{3}(\Phi)=\mathbb{R}$.

Proof of the lemma :

i) To show that $\mathcal{F}_{2}(\Phi)$ (or $\left.\mathcal{F}_{3}(\Phi)\right)$ is a closed subgroup of $(\mathbb{R},+)$, differences with respect to the proof of lemma 3.3 have the following form. Let $y$ and $y^{\prime}$ be in $\mathcal{F}_{2}(\Phi)$ and $\varepsilon>0$. Choose $0<\varepsilon^{\prime}<\varepsilon$ so that $\left|e^{ \pm \varepsilon^{\prime}}-1\right|(|y|+\varepsilon)<\varepsilon$. If for integers $n$ and $m$, one has $\Phi_{m} \in V_{\varepsilon}(0, y, 0)$ and $T^{m} \Phi_{n} \in V_{\varepsilon^{\prime}}\left(0, y^{\prime}, 0\right)$, then $\Phi_{m+n} \in V_{3 \varepsilon}\left(0, y+y^{\prime}, 0\right)$. The rest of the proof is similar.

ii) Analogous modifications of the proof of ergodicity conclude the demonstration.

We now suppose that $\alpha \in[0,1)$, so $\alpha / 2$ is well defined.

THEOREM 4.3.

Let $\alpha$ have non bpq. Choose a strictly increasing sequence $(\theta(n))_{n \geq 1}$ so that $a_{\theta(n+1)+1} \geq 3^{n} q_{\theta(n)}$ and $q_{\theta(n+1)} \geq 3^{n} q_{\theta(n)}^{2}$. Define :

$$
\Phi=\left(\begin{array}{ccc}
e^{f} & 0 & g \\
0 & e^{-f} & h \\
0 & 0 & 1
\end{array}\right)
$$

where:

$$
\left\{\begin{array}{c}
f(x)=\sum_{l \geq 1} \sin \left(\pi q_{\theta(3 l)} \alpha\right) \sin \left[2 \pi q_{\theta(3 l)}(x+\alpha / 2)\right], \\
g(x)=e^{f(x)} \sum_{r \geq 1} \frac{\sin \left(2 \pi q_{\theta(3 r+1)} x\right)}{2^{r} q_{\theta(3 r+1)}} e^{-\frac{1}{2} \sum_{l=1}^{r} \cos \left(2 \pi q_{\theta(3 l)} x\right)} \\
h(x)=e^{-f(x)} \sum_{r \geq 1} \frac{\sin \left(2 \pi q_{\theta(3 r+2)} x\right)}{2^{2} q_{\theta(3 r+2)}} e^{\frac{1}{2} \sum_{l=1}^{r} \cos \left(2 \pi q_{\theta(3 l)} x\right)}
\end{array}\right.
$$

Then $\Phi$ is $\mathcal{C}^{1}$ and $T_{\Phi}: \mathbb{T}^{1} \times \operatorname{Sol}_{3}(\mathbb{R}) \rightarrow \mathbb{T}^{1} \times \operatorname{Sol}_{3}(\mathbb{R})$ is ergodic. 
Remark - Conditions on the sequence $(\theta(n))_{n \geq 1}$ will be seen to be rather rough (as in theorem 3.1). They have the advantage to be easy to present. The same remarks as for the cocycle in theorem 3.1 are valid :

(1) This is an example of $\Phi$ of class $\mathcal{C}^{1}$ such that $\left(\Phi_{n}(x)\right)_{n \geq 0}$ is dense in $\operatorname{Sol}_{3}(\mathbb{R})$ for a full-measure $G_{\delta}$-set of $x$.

(2) There is similar statement for $\alpha$ with bpq and $\Phi$ with a regularity arbitrary close to $\mathcal{C}^{1}$.

(3) Since $\Phi_{n}^{1}=f_{n}$, for all $n \in \mathbb{Z}$, a transformation $T_{\Phi}$ cannot be ergodic when $\alpha$ has bpq and $\Phi$ is $\mathcal{C}^{1}$ or if $\alpha$ has Diophantine type 1 and $\Phi$ is $\mathcal{C}^{1+\varepsilon}$.

We haven't been able to deal with the question of squashability on this example.

\section{Proof of theorem 4.3 :}

Step 1. In the proof, constants are generic. Let us first check that $\Phi$ is $\mathcal{C}^{1}$. For $f$, we have $\sum_{l \geq 1} q_{\theta(3 l)}\left(\left(q_{\theta(3 l)} \alpha\right)\right) \leq C \sum_{l \geq 1} 3^{-3 l}<+\infty$. Concerning $g$ and $h$, this follows from :

$$
\sum_{r \geq 1} 2^{-r} e^{r / 2}\left(1+\frac{\pi r}{q_{\theta(3 r+1)}} \sum_{1 \leq l \leq r} q_{\theta(3 l)}\right)<+\infty
$$

We turn to the ergodicity of $T_{\Phi}$ and wish to apply lemma 4.2 . Since $\Phi_{n}=T^{n-1} \Phi \cdots \Phi$, for $n \geq 1$, when identifying $\operatorname{Sol}_{3}(\mathbb{R})$ to $\mathbb{R}^{3}$ with the composition law in $(4.1)$, we get :

$$
\begin{aligned}
\Phi_{n} & =\left(f_{n}, \sum_{0 \leq k<n}\left(T^{k} g\right) e^{\sum_{k<l<n} T^{l} f}, \sum_{0 \leq k<n}\left(T^{k} h\right) e^{-\sum_{k<l<n} T^{l} f}\right) \\
& =\left(f_{n}, e^{f_{n}} \sum_{0 \leq k<n} T^{k}\left(g e^{-f}\right) e^{-f_{k}}, e^{-f_{n}} \sum_{0 \leq k<n} T^{k}\left(h e^{f}\right) e^{f_{k}}\right) .
\end{aligned}
$$

Consider first $\Phi_{n}^{1}$ and set $t_{n}=\left[\left(a_{\theta(3 n)+1}+1\right) / 2\right] q_{\theta(3 n)}$, for $n \geq 1$. We have :

$$
\Phi_{t_{n}}^{1}(x)=\left(\sum_{l<n}+\sum_{l=n}+\sum_{l>n}\right) \sin \left(\pi q_{\theta(3 l)} t_{n} \alpha\right) \sin \left(\pi q_{\theta(3 l)}\left(2 x+t_{n} \alpha\right)\right)=A_{n}+B_{n}+C_{n}
$$

The extremal terms are estimated by :

$$
\left\{\begin{array}{l}
\left|A_{n}\right| \leq C q_{\theta(3 n-3)}\left(\left(t_{n} \alpha\right)\right) \leq C \frac{q_{\theta(3 n-3)}}{q_{\theta(3 n)}} \rightarrow_{n \rightarrow+\infty} 0 \\
\left|C_{n}\right| \leq C t_{n}\left(\left(q_{\theta(3 n+3)} \alpha\right)\right) \leq C \frac{q_{\theta(3 n+1)}}{q_{\theta(3 n+3)}} \rightarrow_{n \rightarrow+\infty} 0
\end{array}\right.
$$

As a result $\Phi_{t_{n}}^{1}(x)=\sin \left(\pi q_{\theta(3 n)} t_{n} \alpha\right) \sin \left(\pi q_{\theta(3 n)}\left(2 x+t_{n} \alpha\right)\right)+o(1)$, with a uniform $o(1)$. Observe that $\sin \left(\pi q_{\theta(3 n)} t_{n} \alpha\right)=\varepsilon_{n} \sin \left(\pi q_{\theta(3 n)}\left[\left(a_{\theta(3 n)+1}+1\right) / 2\right]\left(\left(q_{\theta(3 n)} \alpha\right)\right)\right)$, where $\varepsilon_{n} \in\{ \pm 1\}$, and :

$$
\frac{1}{4} \leq \frac{a_{\theta(3 n)+1} q_{\theta(3 n)}}{2\left(q_{\theta(3 n)}+q_{\theta(3 n)+1}\right)} \leq q_{\theta(3 n)}\left[\left(a_{\theta(3 n)+1}+1\right) / 2\right]\left(\left(q_{\theta(3 n)} \alpha\right)\right) \leq \frac{\left(a_{\theta(3 n)+1}+1\right) q_{\theta(3 n)}}{2 q_{\theta(3 n)+1}} \leq \frac{3}{4}
$$

for large $n$, since $a_{\theta(3 n)+1} \rightarrow+\infty$. Consequently, there is a subsequence $\left(n^{\prime}\right), \kappa \neq 0$ and $\zeta$ so that along this subsequence :

$$
\Phi_{t_{n^{\prime}}}^{1}(x)=(\kappa+o(1)) \sin \left(2 \pi q_{\theta\left(3 n^{\prime}\right)}(x+\zeta)\right)+o(1),
$$

where each $o(1)$ is uniform. Since $\left(t_{n}\right)_{n \geq 1}$ is a rigid sequence, by lemma 2.2 we deduce that $T_{\Phi^{1}}$ : $\mathbb{T}^{1} \times \mathbb{R} \rightarrow \mathbb{T}^{1} \times \mathbb{R}$ is ergodic. 
Step 2. We now focus on 2-essential values for $\Phi$. Let $u_{n}=2^{n} q_{\theta(3 n+1)}$, for $n \geq 1$. A similar computation gives $f_{u_{n}}(x) \rightarrow 0$, uniformly as $n \rightarrow+\infty$. Consider next :

$$
\Phi_{u_{n}}^{2}(x)=(1+o(1)) \sum_{0 \leq k<u_{n}} T^{k}\left(g e^{-f}\right) e^{-\sum_{l \geq 1} \sin \left(\pi q_{\theta(3 l)} k \alpha\right) \sin \left(\pi q_{\theta(3 l)}(2 x+k \alpha)\right)} .
$$

Set $S_{n}(k)=\sum_{l>n} \sin \left(\pi q_{\theta(3 l)} k \alpha\right) \sin \left(\pi q_{\theta(3 l)}(2 x+k \alpha)\right)$. Clearly $\left|S_{n}(k)\right| \leq C 2^{n} q_{\theta(3 n+1)} / q_{\theta(3 n+3)}$, when $0 \leq k<u_{n}$. As a consequence :

$$
\begin{aligned}
& \left|\sum_{0 \leq k<u_{n}} T^{k}\left(g e^{-f}\right) e^{-\sum_{1 \leq l \leq n} \sin \left(\pi q_{\theta(3 l)} k \alpha\right) \sin \left(\pi q_{\theta(3 l)}(2 x+k \alpha)\right)}\left(e^{-S_{n}(k)}-1\right)\right| \\
& \leq C \frac{\left(2^{n} q_{\theta(3 n+1)}\right)^{2} e^{n}}{q_{\theta(3 n+3)}} \leq C \frac{\left(2^{n} q_{\theta(3 n+1)}\right)^{2} e^{n}}{3^{3 n+3} q_{\theta(3 n+2)}^{2}} \rightarrow_{n \rightarrow+\infty} 0 .
\end{aligned}
$$

Since $\sin \left(\pi q_{\theta(3 l)} k \alpha\right) \sin \left(\pi q_{\theta(3 l)}(2 x+k \alpha)\right)=\left(\cos \left(2 \pi q_{\theta(3 l)} x\right)-\cos \left(2 \pi q_{\theta(3 l)}(x+k \alpha)\right)\right) / 2$, we thus obtain $\Phi_{u_{n}}^{2}=(1+o(1)) \Psi_{n}+o(1)$, where :

$$
\begin{aligned}
\Psi_{n}(x) & =e^{-\frac{1}{2} \sum_{l=1}^{n} \cos \left(2 \pi q_{\theta(3 l)} x\right)} \sum_{k=0}^{u_{n}-1}\left(g e^{-f}\right)(x+k \alpha) e^{\frac{1}{2} \sum_{l=1}^{n} \cos \left(2 \pi q_{\theta(3 l)}(x+k \alpha)\right)} \\
& =e^{-\frac{1}{2} \sum_{l=1}^{n} \cos \left(2 \pi q_{\theta(3 l)} x\right)} \sum_{r \geq 1} \sum_{k=0}^{2^{n} q_{\theta(3 n+1)}-1} T^{k}\left(\frac{\sin \left(2 \pi q_{\theta(3 r+1)} x\right)}{2^{r} q_{\theta(3 r+1)}} e^{\frac{1}{2}\left(\sum_{l=1}^{n}-\sum_{l=1}^{r}\right) \cos \left(2 \pi q_{\theta(3 l)} x\right)}\right) .
\end{aligned}
$$

Decompose $\Psi_{n}=U_{n}+V_{n}+W_{n}$, according to indices $r$ respectively satisfying $r>n, r<n$ and $r=n$. Clearly :

$$
\left|U_{n}\right| \leq C e^{n / 2} \sum_{r>n} \frac{2^{n} q_{\theta(3 n+1)}}{2^{r} q_{\theta(3 r+1)}} e^{r / 2} \leq C e^{n / 2} \frac{q_{\theta(3 n+1)} e^{n / 2}}{3^{3 n} q_{\theta(3 n+2)}^{2}} \rightarrow_{n \rightarrow+\infty} 0 .
$$

Next :

$$
\left|V_{n}\right| \leq C e^{n / 2} \sum_{1 \leq r<n} \frac{1}{2^{r} q_{\theta(3 r+1)}}|X(n, r)|
$$

where :

$$
\begin{aligned}
& X(n, r)=\operatorname{Im} \sum_{k=0}^{2^{n} q_{\theta(3 n+1)}-1} e^{2 i \pi q_{\theta(3 r+1)}(x+k \alpha)} e^{\frac{1}{2} \sum_{l=r+1}^{n} \cos \left(2 \pi q_{\theta(3 l)}(x+k \alpha)\right)} \\
& =\operatorname{Im} \sum_{k=0}^{2^{n} q_{\theta(3 n+1)}-1} e^{2 i \pi q_{\theta(3 r+1)}(x+k \alpha)} e^{\frac{1}{4} \sum_{l=r+1}^{n}\left(e^{2 i \pi q_{\theta(3 l)}(x+k \alpha)}+e^{-2 i \pi q_{\theta(3 l)}(x+k \alpha)}\right)} \\
& =\operatorname{Im} \sum_{s_{l}, t_{l} \geq 0, r<l \leq n} \frac{1}{\prod_{r<l \leq n} 4^{s_{l}+t_{l}} s_{l} ! t_{l} !} \sum_{k=0}^{2^{n} q_{\theta(3 n+1)}-1} e^{2 i \pi\left(q_{\theta(3 r+1)}+\sum_{r<l \leq n}\left(s_{l}-t_{l}\right) q_{\theta(3 l)}\right)(x+k \alpha)} .
\end{aligned}
$$

Set $R_{r, n}\left(\left(s_{l}, t_{l}\right)\right)=q_{\theta(3 r+1)}+\sum_{l=r+1}^{n}\left(s_{l}-t_{l}\right) q_{\theta(3 l)}$. Remark that in the last sum of the right-hand side, a term such that $R_{r, n}\left(\left(s_{l}, t_{l}\right)\right)=0$ does not intervene, since we take the imaginary part.

Decompose next $X(n, r)=X^{1}(n, r)+X^{2}(n, r)$, whether the condition $R_{r, n}\left(\left(s_{l}, t_{l}\right)\right)<q_{\theta(3 n+1)}$ is satisfied or not.

In the first case, we use that $\left(\left(R_{r, n}\left(\left(s_{l}, t_{l}\right)\right) \alpha\right)\right)>\left(\left(q_{\theta(3 n+1)-1} \alpha\right)\right)>1 /\left(2 q_{\theta(3 n+1)}\right)$ and $R_{r, n}\left(\left(s_{l}, t_{l}\right)\right) \leq C q_{\theta(3 n)} \prod_{r<l \leq n}\left(s_{l} \vee 1\right)\left(t_{l} \vee 1\right)$. Since $\sum_{s \geq 0}(s \vee 1) /\left(4^{s} s !\right)=1+e^{1 / 4} / 4$, we obtain : 


$$
\begin{aligned}
\left|X^{1}(n, r)\right| & \leq C\left(\sum_{R_{r, n}\left(\left(s_{l}, t_{l}\right)\right)<q_{\theta(3 n+1)}} \frac{\prod_{r<l \leq n}\left(s_{l} \vee 1\right)\left(t_{l} \vee 1\right)}{\prod_{r<l \leq n} 4^{s_{l}+t_{l}} s_{l} ! t_{l} !}\right) 2^{n}\left(\left(q_{\theta(3 n+1)} \alpha\right)\right) q_{\theta(3 n)} q_{\theta(3 n+1)} . \\
& \leq C 2^{n}\left(1+\frac{e^{1 / 4}}{4}\right)^{n-r} \frac{q_{\theta(3 n)}}{a_{\theta(3 n+1)+1}}
\end{aligned}
$$

In the second case and when $n$ is large enough, there is some $s_{l}$ or $t_{l}$ larger that $q_{\theta(3 n+1)} /\left(n q_{\theta(3 n)}\right)$. Consequently :

$$
\left|X^{2}(n, r)\right| \leq C 2^{n} q_{\theta(3 n+1)} e^{(n-r) / 2} n \sum_{s>q_{\theta(3 n+1)} /\left(n q_{\theta(3 n)}\right)} \frac{1}{4^{s} s !} .
$$

Finally, from (4.3) we obtain :

$$
\begin{aligned}
\left|V_{n}\right| & \leq C(2 \sqrt{e})^{n}\left[\left(1+\frac{e^{1 / 4}}{4}\right)^{n} \frac{q_{\theta(3 n)}}{a_{\theta(3 n+1)+1}}+n q_{\theta(3 n+1)} \sum_{s>q_{\theta(3 n+1)} /\left(n q_{\theta(3 n)}\right)} \frac{1}{4^{s} s !}\right] \\
& \leq C\left(\frac{6^{n}(7 / 4)^{n}}{27^{n}}+6^{n} \frac{q_{\theta(3 n+1)} n^{3} q_{\theta(3 n)}^{2}}{q_{\theta(3 n+1)}^{2}}\right) \rightarrow_{n \rightarrow+\infty} 0 .
\end{aligned}
$$

The conclusion of these calculations is that :

$$
\begin{aligned}
\Phi_{u_{n}}^{2} & =(1+o(1)) e^{-\frac{1}{2} \sum_{l=1}^{n} \cos \left(2 \pi q_{\theta(3 l)} x\right)} \sum_{k=0}^{2^{n} q_{\theta(3 n+1)-1}} \frac{\sin \left(2 \pi q_{\theta(3 n+1)}(x+k \alpha)\right)}{2^{n} q_{\theta(3 n+1)}}+o(1) \\
& =\delta_{n}(1+o(1)) e^{-\frac{1}{2} \sum_{l=1}^{n} \cos \left(2 \pi q_{\theta(3 l)} x\right)} \sin \left(2 \pi q_{\theta(3 n+1)} x\right)+o(1)
\end{aligned}
$$

for some fixed sequence $\delta_{n} \in\{ \pm 1\}$ and uniform $o(1)$. Similar computations give $\Phi_{u_{n}}^{3}=o(1)$, uniformly.

Step 3. We prove that $\mathcal{F}_{2}(\Phi)=\mathbb{R}$. Recall from lemma 4.2 that $\mathcal{F}_{2}(\Phi)$ is a closed subgroup of $(\mathbb{R},+)$. Let $c \in[0,1]$ and suppose that $\sin (2 \pi c) \notin \mathcal{F}_{2}(\Phi)$. Let then $\varepsilon>0$ and $B$ be a Borel set with $\mu(B)>0$ such that :

$$
\mu\left(B \cap T^{-n} B \cap\left\{\Phi_{n} \in V_{\varepsilon}(0, \sin (2 \pi c), 0)\right\}\right)=0, \forall n \in \mathbb{Z} .
$$

Define $B_{y, \eta}=B \cap[y-\eta / 2, y+\eta / 2]$, for $\eta>0$. By lemma 4.4 stated and proved below, the sequence $\left(\sum_{l=1}^{n} \cos \left(2 \pi q_{\theta(3 l)} x\right), \delta_{n} q_{\theta(3 n+1)} x\right)_{n \geq 1}$ is dense in $\mathbb{R} \times \mathbb{T}^{1}$ for almost-every $x$. Since $\mu(B)>$ 0 , take a Lebesgue density point $y_{0} \in B$ and then a sequence $\left(k_{n}\right)=\left(k_{n}\left(y_{0}\right)\right)$ verifying the conditions :

$$
\frac{\mu\left(B_{y_{0}, \eta}\right)}{\eta} \rightarrow_{\eta \rightarrow 0} 1 \text { and }\left(\sum_{1 \leq l \leq k_{n}} \cos \left(2 \pi q_{\theta(3 l)} x\right), \delta_{n} q_{\theta\left(3 k_{n}+1\right)} x\right) \rightarrow_{n \rightarrow+\infty}(0, c) \in \mathbb{R} \times \mathbb{T}^{1}
$$

For all $n \geq 1$ and $\eta>0$, we get from (4.5) :

$$
\mu\left(B_{y_{0}, \eta} \cap T^{-u_{k_{n}}} B_{y_{0}, \eta} \cap\left\{\Phi_{u_{k_{n}}} \in V_{\varepsilon}(0, \sin (2 \pi c), 0)\right\}\right)=0 .
$$

Set $\eta_{n}=n^{-1 / 2} q_{\theta\left(3 u_{k_{n}}+1\right)}^{-1}$. Via $(4.4), \Phi_{u_{k_{n}}} \rightarrow(0, \sin (2 \pi c), 0)$, uniformly on $B_{y_{0}, \eta_{n}}$. Consequently :

$$
B_{y_{0}, \eta_{n}} \subset\left\{\Phi_{u_{k_{n}}} \in V_{\varepsilon}(0, \sin (2 \pi c), 0)\right\}
$$


for $n$ large enough. Thus $\mu\left(B_{y_{0}, \eta_{n}} \cap T^{-u_{k_{n}}} B_{y_{0}, \eta_{n}}\right)=0$, for large $n$. Clearly, we have the inequality $\left(\left(u_{k_{n}} \alpha\right)\right)<1 /\left(n q_{\theta\left(3 u_{k_{n}}+1\right)}\right)$. Using $\mu(A \cap B)=\mu(A)+\mu(B)-\mu(A \cup B)$, a contradiction is then :

$$
\begin{aligned}
\mu\left(B_{y_{0}, \eta_{n}} \cap T^{-u_{k_{n}}} B_{y_{0}, \eta_{n}}\right) & \geq 2 \eta_{n}(1+o(1))-\left(\eta_{n}+\left(\left(u_{k_{n}} \alpha\right)\right)\right) \\
& \geq q_{\theta\left(3 u_{k_{n}}+1\right)}^{-1}\left(\frac{1}{n^{1 / 2}}(1+o(1))-\frac{1}{n}\right)>0,
\end{aligned}
$$

for $n$ large enough.

This proves that $\sin (2 \pi c) \in \mathcal{F}_{2}(\Phi)$. Since this is true for all $c$, we get $\mathcal{F}_{2}(\Phi)=\mathbb{R}$. The treatment of $\mathcal{F}_{3}(\Phi)$ is symmetric, using this time the sequence $\left(2^{n} q_{\theta(3 n+2}\right)_{n \geq 1}$, giving also $\mathcal{F}_{3}(\Phi)=\mathbb{R}$. By Step 1 and lemma 4.2, we conclude that $T_{\Phi}$ is ergodic. This ends the proof of the theorem.

When proving theorem 4.3, we have used the following result, which does not exactly enter classical schemes. We give an elementary proof.

\section{LEMMA 4.4.}

Let $\left(\delta_{n}\right)_{n \geq 1}$ be a sequence such that $\delta_{n} \in\{ \pm 1\}$ for all $n$. Then $\left(\sum_{l=1}^{n} \cos \left(2 \pi q_{\theta(3 l)} x\right), \delta_{n} q_{\theta(3 n+1)} x\right)_{n \geq 1}$ is dense in $\mathbb{R} \times \mathbb{T}^{1}$ for almost-every $x$.

\section{Proof of the lemma :}

We only use that $\sum_{l \geq 1} q_{\theta(l)} / q_{\theta(l+1)}<+\infty$. Set $X_{l}(x)=\cos \left(2 \pi q_{\theta(3 l)} x\right)$ and $S_{n}=X_{1}+\cdots+X_{n}$. The $\left(X_{n}\right)$ are asymptotically $i i d$, but do not form a stationary process.

A first property is that $S_{n} / \sqrt{n}$ converges in law to $\mathcal{N}_{0,1 / 2}$, as $n \rightarrow+\infty$. Introduce the characteristic function $\varphi(t)=\mathbb{E}\left(e^{i t X_{1}}\right), t \in \mathbb{R}$, where $\mathbb{E}$ is for the expectation under $\mu$. Fix $t \in \mathbb{R}$.

We consider the partition $\mathcal{F}_{n}=\sigma\left\{\left[k / q_{\theta(3 n)},(k+1) / q_{\theta(3 n)}\right), \quad 0 \leq k<q_{\theta(3 n)}\right\}$. Since $\left|S_{n}^{\prime}\right|=$ $O\left(q_{\theta(3 n)}\right)$, uniformly in $n$, we have for some constant $C$ independent of $t$ and $n$ :

$$
\left|e^{i t S_{n}}-\mathbb{E}\left[e^{i t S_{n}} \mid \mathcal{F}_{n+1}\right]\right| \leq C|t| \frac{q_{\theta(3 n)}}{q_{\theta(3(n+1))}} .
$$

Using repeatedly this inequality, we obtain, with a uniform $O($.$) :$

$$
\begin{aligned}
\mathbb{E}\left[e^{\frac{i t}{\sqrt{n}} S_{n}}\right] & =\mathbb{E}\left[\mathbb{E}\left[e^{\frac{i t}{\sqrt{n}} S_{n-1}} \mid \mathcal{F}_{n}\right] e^{\frac{i t}{\sqrt{n}} X_{n}}\right]+O\left(\frac{q_{\theta(3(n-1))}}{\sqrt{n} q_{\theta(3 n)}}\right) \\
& =\mathbb{E}\left[\mathbb{E}\left[e^{\frac{i t}{\sqrt{n}} S_{n-1}} \mid \mathcal{F}_{n}\right] \mathbb{E}\left[e^{\frac{i t}{\sqrt{n}} X_{n}} \mid \mathcal{F}_{n}\right]\right]+O\left(\frac{q_{\theta(3(n-1))}}{\sqrt{n} q_{\theta(3 n)}}\right) \\
& =\varphi(t / \sqrt{n}) \mathbb{E}\left(e^{\frac{i t}{\sqrt{n}} S_{n-1}}\right)+O\left(\frac{q_{\theta(3(n-1))}}{\sqrt{n} q_{\theta(3 n)}}\right) . \\
& =\varphi(t / \sqrt{n})^{n}+O\left(\frac{1}{\sqrt{n}} \sum_{0 \leq l<n} \frac{q_{\theta(3 l)}}{q_{\theta(3(l+1))}}\right) \longrightarrow_{n \rightarrow+\infty} e^{-t^{2} / 4},
\end{aligned}
$$

when setting $q_{\theta(0)}=1$. This proves the first assertion.

We next show that $\liminf S_{n}(x)=-\infty$ and $\lim \sup S_{n}(x)=+\infty$ for almost every $x$. Define $M(x)=\sup _{n} S_{n}(x)$. Since $\left|X_{l}(x)-X_{l}(T x)\right| \leq C\left(\left(q_{\theta(3 l)} \alpha\right)\right)$ and this quantity is summable in $l$, the set $\{M(x)<+\infty\}$ is $T$-invariant and thus has measure zero or one. In the second case, we get $\mu(M(x) \geq n) \rightarrow 0$, as $n \rightarrow+\infty$, but this contradicts the CLT, because :

$$
\mu(M(x) \geq n) \geq \mu\left(S_{n^{2}} \geq n\right) \rightarrow_{n \rightarrow+\infty} \mathcal{N}_{0,1 / 2}[1,+\infty)>0 .
$$

As a result, $\lim \sup S_{n}(x)=+\infty$ almost surely. Idem, $\lim \inf S_{n}(x)=-\infty$ almost surely. 
Suppose by contradiction that the result is not true. The separability of $\mathbb{R} \times \mathbb{T}^{1}$ implies that there exist $(a, b) \in \mathbb{R} \times \mathbb{T}^{1}, \varepsilon>0$ and a Borel set $A \subset \mathbb{T}^{1}$ with $\mu(A)>0$ such that for $x \in A$, $\left(S_{n}(x), \delta_{n} q_{\theta(3 n+1)} x\right) \notin V_{\varepsilon}(a, b)$, for all $n \geq 0$.

Using the previous remarks, denote for almost-every $x$ by $\left(\tau_{n}(x)\right)_{n \geq 1}$ the random infinite sequence so that $\left|S_{\tau_{n}(x)}(x)-a\right|<1 / 2$. Choose a density point $x_{0}$ in $A$ with such an associated sequence.

Since $\left|S_{k}^{\prime}(x)\right| \leq 2 \pi \sum_{l=1}^{k} q_{\theta(3 l)}=o\left(q_{\theta(3(k+1))}\right)$, as $k \rightarrow+\infty$, in the ball $B\left(x_{0}, 1 / q_{\theta\left(3\left(\tau_{n}\left(x_{0}\right)+1\right)\right)}\right)$ the set of $x$ such that $S_{\tau_{n}\left(x_{0}\right)+1}(x) \in V_{\varepsilon / 2}(a)$ contains an interval of length $C \varepsilon / q_{\theta\left(3\left(\tau_{n}\left(x_{0}\right)+1\right)\right)}$, for some constant $C>0$. On this interval, the proportion of $x$ such that, modulo one, $\delta_{n} q_{\theta\left(3\left(\tau_{n}\left(x_{0}\right)+1\right)+1\right)} x \in$ $V_{\varepsilon / 2}(c)$ is larger than $C^{\prime} \varepsilon$, for another constant $C^{\prime}>0$.

Consequently, a proportion at least $C C^{\prime} \varepsilon^{2}$ of $x$ in $B\left(x_{0}, 1 / q_{\theta\left(3\left(\tau_{n}\left(x_{0}\right)+1\right)\right)}\right)$ have the property that $\left(S_{n}(x), \delta_{n} q_{\theta(3 n+1)} x\right) \in V_{\varepsilon}(a, b)$ for some $n \geq 0$. This contradicts the fact that $x_{0}$ is a density point in $A$ and concludes the proof of the lemma.

Acknowledgements. We thank Jean-Pierre Conze for discussions on cocycles and Benoît Daniel for mentioning reference [19].

\section{References}

1. Aaronson, J., An introduction to infinite ergodic theory, Mathematical surveys and monographs 50, AMS, Providence, 1997.

2. Aaronson, J., The asymptotic distributional behaviour of transformations preserving infinite measures, J. Anal. Math. 39 (1981), 203-234.

3. Arnold, V., Small denominators. I. Mapping the circle onto itself, Izv. Akad. Nauk SSSR Ser. Mat. 25 (1961), $21-86$.

4. Conze, J.-P., Recurrence, ergodicity and invariant measures for cocycles over a rotation, preprint 2007.

5. Conze, J.-P. ANd Chevallier, N., Examples of recurrent or transient stationary walks in $\mathbb{R}^{d}$ over a rotation of $\mathbb{T}^{2}$, preprint 2008 .

6. FAYAD, B., On the ergodicity of the Weyl sums cocycles, Acta Arith. 125 (2006), 305-316.

7. Feldman, J. And Moore, C., Ergodic equivalence relations, cohomology and von Neumann algebras, I. Trans. Amer. Math. Soc. 234 (1977), no.2, 289-324.

8. Gelbrich, G.., Self-similar periodic tilings on the Heisenberg group, Journal of Lie Theory, Vol. 4 (1994), 31-37.

9. Golodets, V. And Sinel'shchikov, S., Locally compact groups appearing as ranges of cocycles of ergodic $\mathbb{Z}$-actions, Ergodic Theory and Dynamical Systems 5 (1985), 45-57.

10. Greschonig, G., Recurrence in unipotent groups and ergodic nonabelian group extensions, Israël Journal of Math. vol. 147, 245-267, 2005.

11. Herman, M., Sur la conjugaison différentiable des difféomorphismes du cercle à des rotations, Inst. Hautes Etudes Sci. Publ. Math. No. 49 (1979), 5-233.

12. Kuipers, L. And Niederreiter, H., Uniform distribution of sequences. Pure and applied mathematics., WileyInterscience, New York-London-Sydney, 1974

13. Lemańczyk, M., Parreau, F. and Volný, D., Ergodic properties of real cocycles and pseudo-homogeneous Banach spaces, Trans. of the AMS, vol. 348, no. 12, 1996, 4919-4938.

14. Liardet, P. And Volný, D., Sums of continuous and differentiable functions in dynamical systems, Israël Journal of Math. vol. 98, 29-60, 1997.

15. Nerurkar, M., Ergodic continuous skew product actions of amenable groups, Pacific J. Math. 119 (1985), no. $2,343-363$.

16. Nerurkar, M., On the construction of smooth ergodic skew-products, Ergodic Theory and Dynamical Systems 8 (1988), no. 2, 311-326.

17. Schmidt, K., Lectures on Cocycles of Ergodic Transformations Groups, Lecture Notes in Math. vol. 1, Mac Millan Co. of India (1977).

18. Thurston, W., Three dimensional geometry and topology, Princeton University press, 1997.

19. Troyanov, M., L'horizon de Sol, Exposition. Math. 16 (1998), no. 5, 441-479.

20. Volný, D., Completely squashable smooth ergodic cocycles over irrational rotations, Topol. Methods Nonlinear Anal. 22 (2003), no. 2, 331-344.

21. Yoccoz, J.-C., Sur la disparition de propriétés de type Denjoy-Koksma en dimension 2, C. R. Acad. Sci. Paris Sér. A-B 291 (1980), no. 13.

Laboratoire d’Analyse et de Mathématiques Appliquées, Université Paris XiI, Faculté des Sciences et Technologies, 61 avenue du Général de Gaulle, 94010 Créteil Cedex, FranCE E-mail address : bremont@univ-paris12.fr 